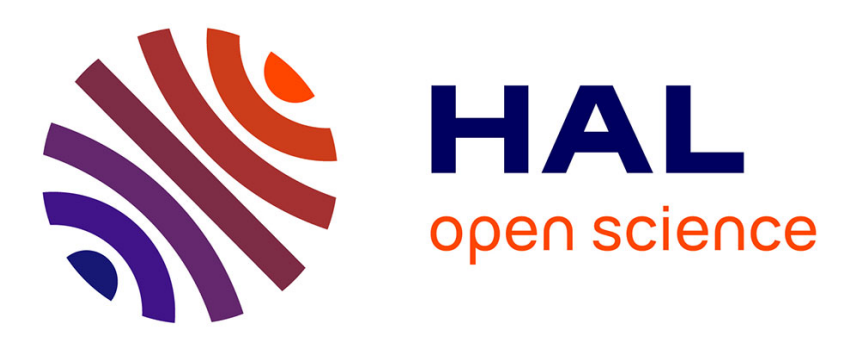

\title{
A Novel Task-Based Reconstruction Approach for Digital Breast Tomosynthesis
}

Maissa Sghaier, Emilie Chouzenoux, Jean-Christophe Pesquet, Serge Muller

\section{To cite this version:}

Maissa Sghaier, Emilie Chouzenoux, Jean-Christophe Pesquet, Serge Muller. A Novel Task-Based Reconstruction Approach for Digital Breast Tomosynthesis. Medical Image Analysis, 2022, 77, pp.102341. 10.1016/j.media.2021.102341 . hal-02972386v2

\section{HAL Id: hal-02972386 \\ https://hal.science/hal-02972386v2}

Submitted on 16 Dec 2021

HAL is a multi-disciplinary open access archive for the deposit and dissemination of scientific research documents, whether they are published or not. The documents may come from teaching and research institutions in France or abroad, or from public or private research centers.
L'archive ouverte pluridisciplinaire HAL, est destinée au dépôt et à la diffusion de documents scientifiques de niveau recherche, publiés ou non, émanant des établissements d'enseignement et de recherche français ou étrangers, des laboratoires publics ou privés. 


\title{
A Novel Task-Based Reconstruction Approach for Digital Breast Tomosynthesis
}

\author{
Maissa Sghaier $^{\mathrm{a}, \mathrm{b}, *}$, Emilie Chouzenoux ${ }^{\mathrm{a}, 1}$, Jean-Christophe Pesquet ${ }^{\mathrm{a}, 2}$, Serge Muller ${ }^{\mathrm{b}, 3}$ \\ ${ }^{a}$ University of Paris-Saclay, CentraleSupélec, CVN, Inria, 09 Rue Joliot Curie, Gif-sur-Yvette 91190, France \\ ${ }^{b}$ GE Healthcare, 283 Rue de la Minière, Buc 78530, France
}

\section{A R T ICLE INFO}

\section{Article history:}

Received XXXX

Received in final form XXXX

Accepted XXXX

Available online XXXX

Communicated by XXXX

Keywords: 3D image reconstruction, Detectability, Digital Breast Tomosynthesis, Inverse problem, Majorize-Minimize Memory Gradient Algorithm, Optimization, Spatially adaptive regularization, Total variation

\begin{abstract}
A B S T R A C T
The reconstruction of a volumetric image from Digital Breast Tomosynthesis (DBT) measurements is an ill-posed inverse problem, for which existing iterative regularized approaches can provide a good solution. However, the clinical task is somehow omitted in the derivation of those techniques, although it plays a primary role in the radiologist diagnosis. In this work, we address this issue by introducing a novel variational formulation for DBT reconstruction, tailored for a specific clinical task, namely the detection of microcalcifications. Our method aims at simultaneously enhancing the detectability performance and enabling a high-quality restoration of the background breast tissues. Our contribution is threefold. First, we introduce an original task-based reconstruction framework through the proposition of a detectability function inspired from mathematical model observers. Second, we propose a novel total-variation regularizer where the gradient field accounts for the different morphological contents of the imaged breast. Third, we integrate the two developed measures into a cost function, minimized thanks to a new form of the Majorize Minimize Memory Gradient (3MG) algorithm. We conduct a numerical comparison of the convergence speed of the proposed method with those of standard convex optimization algorithms. Experimental results show the interest of our DBT reconstruction approach, qualitatively and quantitatively.
\end{abstract}

(C) 2021 Elsevier B. V. All rights reserved.

\section{Introduction}

Nowadays, breast cancer is the most prominent cancer among women and the second leading cause of death among them. According to the World Health Organization, it impacts 2.1 millions of women each year. Early screening and diagnosis can make a life-changing shift in the mortality rates. For years, full field digital mammography (FFDM) has been the gold standard for screening and early detection of breast cancer. It has significantly reduced breast cancer mortality in a cost-effective way. Nevertheless, as a 2D imaging technique its sensitivity is affected by breast tissue overlapping Kolb et al. (2002), in a particularly severe way for highly dense breasts.

\footnotetext{
*Corresponding author: Tel.: +33-615-328-307;

e-mail: maissasghaier@gmail.com (Maissa Sghaier)

1 emilie.chouzenoux@centralesupelec.fr

2 jean-christophe@pesquet.eu

3 serge.muller@ge.com
} 
Digital Breast Tomosynthesis (DBT) was introduced to partially alleviate the major limitation of FFDM, induced by the tissue overlap Sechopoulos (2013a). The acquisition geometry is very similar to that used in mammography. However, in DBT, several projections of the breast are used for the reconstruction of a stack of images parallel to the detector plane, corresponding to breast slices. A limited number of low-dose projection images is acquired so that the total dose to the patient in DBT remains comparable to the one in FFDM. From the acquired DBT two-dimensional projection images, the sought three-dimensional volume is computed, using a reconstruction algorithm. The resulting volume consists of slices that present less superimposition of tissues compared to a standard X-ray mammography. Hence, thanks to this 3D nature of the reconstructed volume, DBT offers a great potential to help reducing recall rates, to improve the accuracy of breast cancer detection, and therefore to improve the clinical diagnosis performance, particularly for dense breasts.

The diagnosis accuracy in breast cancer screening highly depends on the ease for the radiologist to detect microcalcifications within the analysed images. The detectability of microcalcifications remains an open challenge in the context of DBT, as emphasized in many recent research studies Spangler et al. (2011); Michell et al. (2012); Poplack et al. (2007); Kopans et al. (2011); Destounis et al. (2013); Li et al. (2018a); Horvat et al. (2019); Kuwabara et al. (2020). First, microcalcifications detection is a particularly challenging task due to their small size and the fact of potential occlusion of those objects in highly dense breasts. Second, the high number of images to be reviewed in DBT (3D scans in DBT versus 2D images in FFDM) increases the time and complexity of the interpretation for the radiologist, which may negatively affect his/her performance in terms of visual detection accuracy. Third, due to inherent geometric limitations, the reconstructed DBT volumes remain characterized by anisotropic spatial resolution, with high resolution in the planes parallel to the detector and much lower resolution in the perpendicular direction.

One promising research direction to enhance microcalcification detectability performance in DBT is to investigate over the reconstruction process of DBT volumes. As DBT volume reconstruction is an ill-posed inverse problem, iterative reconstruction algorithms have demonstrated their superiority over analytical ones Sechopoulos (2013b). In particular, regularized iterative algorithms have the potential to incorporate prior knowledge aiming at mitigating the missing information issue Sidky et al. (2007. 2009); Metin et al. (2014); Piccolomini and Morotti (2016); Szasz et al. (2016); Rose et al. (2017); Dang et al. (2017). Even though these regularized approaches yield smoother DBT reconstructed volumes, they do not account for the aforementioned clinical task, i.e. microcalcification detection. Furthermore, it is well known that the breast is composed by several anatomical components with very different attenuation properties compared with the ones of the microcalcifications. Resorting to classical regularization approaches, such as the celebrated total-variation (TV), may not be enough to cope with such heterogeneity within the DBT images.

In this work, we introduce a novel variational formulation for DBT reconstruction which is specifically adapted to the targeted clinical task. Our contribution is threefold. First, we formulate a new detectability function as a prior term aiming at maximizing the detectability of microcalcifications in predefined regions of interest. Second, we introduce a novel TV-based regularization strategy, involving an original discretization of the gradient field of the image that incorporates prior knowledge on the morphological contents of our image. Third, we propose an efficient algorithm, based on the Majorize-Minimize Memory Gradient (3MG) scheme, to minimize the resulting objective function. Numerical experiments, conducted on phantom and clinical data, assess qualitatively the performance on the proposed reconstruction approach. Finally, a visual evaluation study, performed on fourteen qualified readers demonstrates the interest of our proposed approach.

The paper is organized as follows: Section 2 introduces the reconstruction problem and motivates our proposed approach. Section 3 presents our proposed task-based approach, relying on the formulation of a novel microcalcification detectability term. Section 4 introduces the new spatially adaptive total variation (SATV) regularization term. Section 5 depicts the application of $3 \mathrm{MG}$ 
algorithm, including several numerical improvements tailored to our DBT reconstruction problem. The conducted experimental results are presented in Section 6 . Section 7 concludes this work.

\section{Reconstruction problem in DBT}

\subsection{Problem statement}

The objective of DBT reconstruction is to recover a volumetric estimation of the imaged object from a limited set of measurements. Those are linked to the imaged object through a linear forward projector, so that

$$
p=A d+e
$$

where $p \in \mathbb{R}^{n}$ represents the $n \geq 1$ acquired projections data, $d=\left(d_{i}\right)_{1 \leq i \leq m} \in \mathbb{R}^{m}$ represents the DBT volume of $m \geq 1$ voxels to be reconstructed, $A \in \mathbb{R}^{n \times m}$ is the forward projection matrix describing the geometry of the DBT system, and $e \in \mathbb{R}^{n}$ is the noise. Mathematically, DBT reconstruction is a linear inverse problem. Under zero-mean white Gaussian noise assumptions, the maximum likelihood solution leads to the minimization of the least squares cost function

$$
\left(\forall d \in \mathbb{R}^{m}\right) \quad f(d)=\frac{1}{2}\|p-A d\|_{2}^{2}
$$

However, the minimization of (2) is likely to provide a poor quality DBT reconstruction. This is due to the ill-conditioning of the projection matrix $A$. The noise tends to be amplified even if present in a small amount in the input projection data. The key is to regularize the problem so that the solution is less sensitive to perturbations. A common efficient regularization strategy consists in minimizing a penalized least squares function, that is

$$
\underset{d \in \mathbb{R}^{m}}{\operatorname{minimize}} \frac{1}{2}\|p-A d\|_{2}^{2}+\varphi(d)
$$

where $\varphi: \mathbb{R}^{m} \rightarrow \mathbb{R}$ denotes a regularization function that aims at improving the robustness to noise Sechopoulos 2013b); Rose et al. (2019, 2017); Dang et al. (2017). Herein, $\varphi$ can take several possible forms to enforce spatial regularity, range constraints, or sparsity in a possibly transformed domain Pustelnik et al. (2016). In essence, it can be any function that encodes prior knowledge on the solution.

\subsection{Proposed task-based regularization}

The detection of microcalcifications represents a challenge in DBT Spangler et al. (2011). Since DBT is not yet approved as stand-alone, DBT exam typically combines reviewing DBT and 2D mammography. Interpreting a DBT volume requires sifting through and analysing the DBT image slices whose number depends on the breast thickness. The time spent by the radiologist is higher than for the FFDM exam since there are more images to be reviewed. It is thus of main importance to ease the microcalcifications detection task for the radiologist, while reconstructing DBT volumes to mitigate practical error rates.

To this aim, the DBT reconstructed volume should not only be regular but also account for the image review workflow and thus for the morphological content of the imaged breast. The breast is composed by several anatomical components which have different attenuation properties than the microcalcifications. The DBT reconstructed volume should not only be regular but also account for the image review workflow and thus for the morphological content of the imaged breast. Resorting to standard image regularization techniques may not be enough to meet with this specificity. In this work, we propose an original formulation of the penalty function $\varphi$ in the minimization problem (3), taking the form: 


$$
\left(\forall d \in \mathbb{R}^{m}\right) \quad \varphi(d)=-\alpha D(d)+\Psi(L d)+\frac{\gamma}{2}\|d\|_{2}^{2}+\kappa Q(d)
$$

Hereabove, $D: \mathbb{R}^{m} \rightarrow \mathbb{R}$ denotes the detectability function with weight $\alpha \geq 0$, that will be tailored to enhance the visibility of microcalcifications in some pre-defined regions of interest. In Section 3.1, we will show that a linear form for $D$ can be chosen. The minus sign in front of $\alpha$ comes from to the fact that high values of $D$ indicate the presence of microcalcifications. Moreover, $\Psi$ represents a sparsity promoting function and $L$ is a linear operator from $\mathbb{R}^{m}$ to $\mathbb{R}^{3 m}$ allowing us to select locally either the image intensity or its gradients (see details in Section 4). This spatially adaptive total variation (SATV) regularization term will be constructed with the aim to enable a more adequate spatial regularization in the DBT volume accounting for some prior knowledge on the various regions of the volume. An elastic net regularization term $\|\cdot\|_{2}^{2}$ with weight $\gamma \geq 0$ is included to ensure the uniqueness of the solution. Finally, the values of the reconstructed volume are encouraged to lie in a specific range of values $\left[0, d_{\text {max }}\right]$, with $d_{\max }>0$, through the introduction of the penalty function $Q$ scaled with $\kappa>0$, that reads

$$
\left(\forall d=\left(d_{i}\right)_{1 \leq i \leq m} \in \mathbb{R}^{m}\right) \quad Q(d)=\sum_{i=1}^{m} \inf _{a \in\left[0, d_{\max }\right]}\left(d_{i}-a\right)^{2} .
$$

It is worth mentioning that, when $\kappa$ goes to infinity, $\kappa Q$ tends to the indicator function for the convex domain $\left[0, d_{\text {max }}\right]^{m}$.

We will describe in a detailed manner the construction of functions $D$ and $\Phi$ in Sections 3 and 4 Then, Section 5 will be dedicated to the proposition of an efficient iterative algorithm for the resolution of (3)-(4).

\section{Construction of the detectability function}

We present in this section the mathematical definition of the proposed detectability function $D$ in (4). The aim of this term is to adapt the reconstruction procedure in order to help the radiologist in the microcalcification detection task. A typical workflow that involves the latter task in DBT, includes all the steps from the data acquisition step to the detection task at the very final step (Figure 11). Once the DBT volume is reconstructed and post-processed, if needed, the radiologist searches over regions of interest within the DBT volume with multiple eye movements looking for highlighted structures. Then, he/she decides whether a cluster of microcalcifications is present or absent (yes/no decision). Nonetheless, there are several downsides when performing these different steps independently. Within this configuration, the reconstruction step does not account for the microcalcification detection task in the sense that the latter task is performed in an independent manner by the radiologist at the very final step.

It is thenceforth natural to be raise the question of how to integrate the microcalcification detectability task in the reconstruction process so as to improve the radiological performance for this clinical task. This work will provide a potential answer by proposing a new DBT reconstruction approach that is designed in order to maximize the visibility of microcalcifications. The microcalcifications detectability task can be efficiently assessed by using anthropomorphic model observers Van Metter et al. (2000); Platiša et al. (2011). Anthropomorphic model observers refer to mathematical observer models that were developed to assess image quality for a specific clinical task Barrett et al.(1993). These observers mimic the human observer for such a task, in contrast with ideal observers (IOs) which assume a full knowledge of the statistics of the image Park et al. (2009). In essence, by applying an anthropomorphic model observer to a given image, one would obtain as an output a scalar decision variable that determines how detectable is the lesion for the radiologist. Here, we propose to introduce a new penalty function in our reconstruction method whose formulation takes its roots into the framework of anthropomorphic model observers. The construction of this novel function, acting as an enhancer for the microcalcifications detectability, is explained hereafter. 


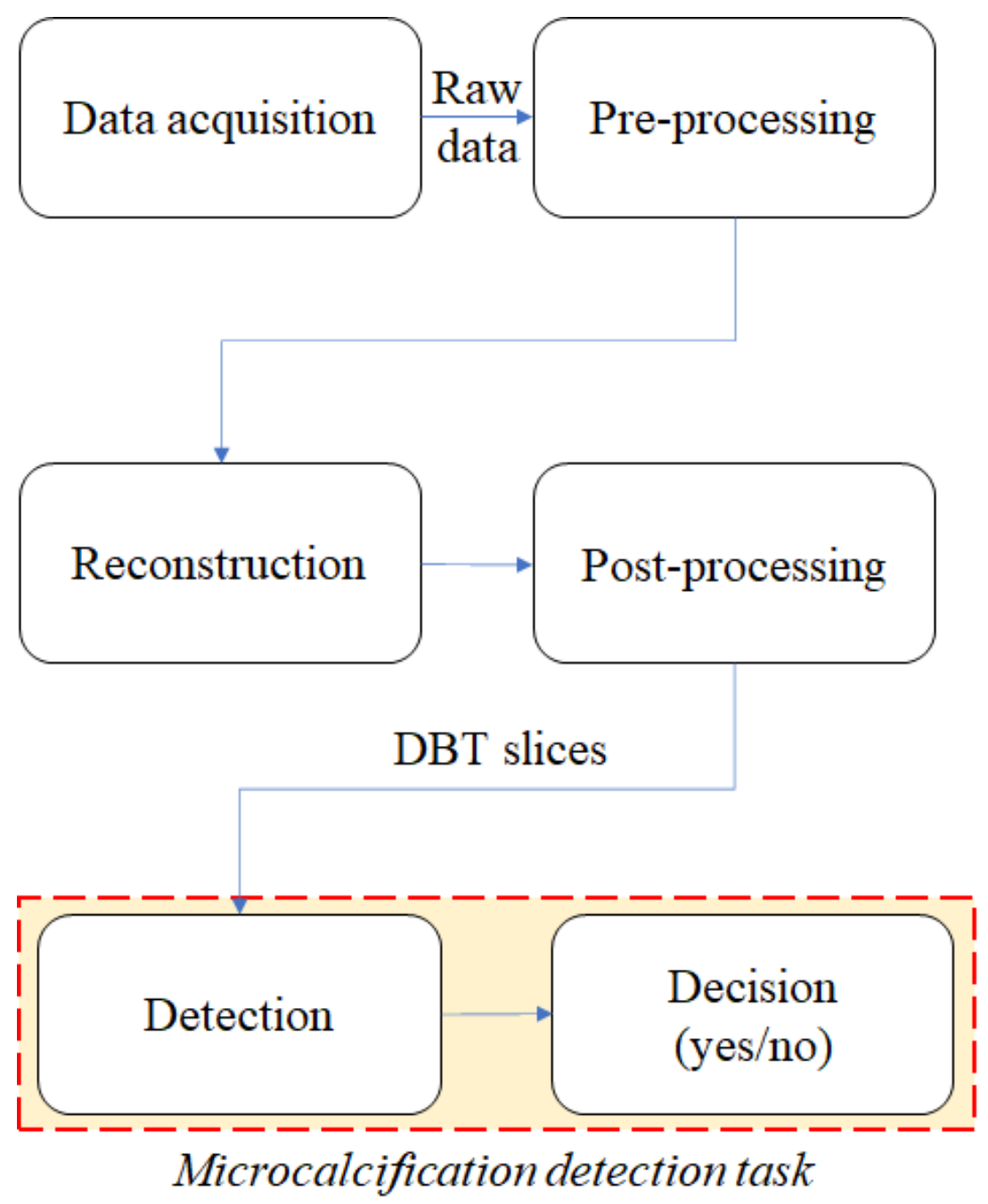

Fig. 1: A schematic of a typical worflow involving microcalcification detection task in DBT. In the first row, raw data is acquired and pre-processed. In the second row, the pre-processed data is used as input to a reconstruction algorithm then potentially post-processed, if needed in the aim of delivering a DBT volume with a certain image quality. The final outcome will be used to perform the detection task presented under the dotted rectangular part. The latter part outlines the steps of microcalcification detection task performed by the radiologist in DBT.

\subsection{Introduction of an anthropomorphic model observer}

Let us consider a set of $q$ vectors $\left(r^{i}\right)_{1 \leq i \leq q}$ of intensity values in regions of interest (ROIs) where, for every $i \in\{1, \ldots, q\}, r^{i} \in \mathbb{R}^{k}$ with $k<m$ is the vector defining a zone where a microcalcification is potentially present. For every $i \in\{1, \ldots, q\}$, let us introduce the decimation operator $S_{i}$ that extracts a region of interest $r^{i}$ from a given volume $d \in \mathbb{R}^{m}$ as follows:

$$
\begin{aligned}
S_{i}: \mathbb{R}^{m} & \rightarrow \mathbb{R}^{k} \\
d & \mapsto r^{i}=\left(d_{j}\right)_{j \in \mathbb{I}^{i}},
\end{aligned}
$$

where each $\mathbb{I}^{i}$ denotes the set of indices of $k$ voxels related to each region of interest $r^{i}$. For simplicity, we will assume that these regions do not overlap, i.e., $\forall(i, j) \in\{1, \ldots, q\}^{2}, \mathbb{I}^{i} \cap \mathbb{I}^{j}=\varnothing$ if $i \neq j$. Following the approach used in Channelized Hotelling observer (CHO) Platiša et al. (2011), itself an extension of Hotelling observer (HO) Barrett et al. (1993), each $r^{i}$ is channelized into $c$ discriminative features through a decomposition matrix $U \in \mathbb{R}^{k \times c}$ where each column denotes a single channel with size equal to the one of the original region of interest. Thereby, we obtain a feature vector $v^{i}=U^{\top} r^{i}$. The channelization process is crucial for two reasons. First, it allows to simplify the computation complexity in HO by greatly reducing the dimension of the input 
images when $c \ll k$ Myers and Barrett (1987). Second, it was demonstrated that the channelization mechanism enables to model characteristics of the human visual system in certain conditions by choosing adequate channel filters Park et al. (2007); Van Metter et al. (2000); Henrot et al. (2014).

Let us consider a ROI index $i \in\{1, \ldots, q\}$, and associated vectors $r^{i}$ and $v^{i}$. The detection task is formulated as a binary hypothesis test where $\mathcal{H}_{0}$ denotes microcalcification-free hypothesis and $\mathcal{H}_{1}$ is microcalcification-presence hypothesis:

$$
\begin{cases}\mathcal{H}_{0}: & r^{i}=b^{i}+\gamma^{i} \\ \mathcal{H}_{1}: & r^{i}=\mu^{i}+b^{i}+\gamma^{i}\end{cases}
$$

where $b^{i}, \mu^{i}$, and $\gamma^{i}$ are real-valued variables which model the background, the signal of interest (i.e., microcalcification) and the noise, respectively. Under the assumption of a zero-mean multivariate Gaussian noise, we show in Appendix A that a natural choice for the detection function is

$$
\left(\forall d \in \mathbb{R}^{m}\right) \quad D(d)=\mu^{\top} \sum_{i=1}^{q} S_{i}^{\top} U \Sigma^{-1} U^{\top} S_{i} d,
$$

where

$$
\mu=\sum_{i=1}^{q} S_{i}^{\top} \mu^{i}
$$

and $\Sigma$ denotes the covariance matrix of the channelized noise. The last equality has been derived from the properties of the operators $S_{i}$ and the assumption that the ROIs do not overlap. Since our goal is to improve the detection of microcalcifications, and $D$ has been built to take higher values when those are present, $D(d)$ is multiplied by a negative factor $(-\alpha)$ to define the detectability term in the objective function. In practice, the ROIs location as well as the signal map $\mu$ will be derived from an intermediate reconstructed volume $d^{*}$ as detailed next.

\subsection{Computer-aided microcalcification detection}

The evaluation of function (8) requires the setting of (i) the ROIs determining the location of potential microcalcifications, (ii) the signal of interest $\mu$ and the associated covariance matrix $\Sigma$. The covariance matrix $\Sigma$ will be learnt using training datasets as described in Section 3.1 The construction of the signal of interest with the associated regions of interest will be based on the postprocessing of a total-variation-based reconstructed volume, followed by a Computer-Aided Detection methodology Christoyianni et al. (2002).

\subsubsection{Signal estimation}

We first solve the following constrained optimization problem:

$$
\underset{\substack{d \in\left[0,+\infty r^{m} \\ \Delta d \in B_{1,2}(0, \zeta)\right.}}{\operatorname{minimize}} \frac{1}{2}\|p-A d\|_{2}^{2},
$$

where $\Delta \in \mathbb{R}^{3 m \times m}$ is the discretized gradient operator along the 3 spatial dimensions,

$$
B_{1,2}(0, \zeta)=\left\{\delta \in \mathbb{R}^{3 m} \mid\|\delta\|_{1,2} \leq \zeta\right\},
$$

$\|\cdot\|_{1,2}$ denotes the $\ell_{1,2}$ norm, and $\zeta>0$ is a preset constraint bound on the total-variation of the volume.

The objective function in Problem 10 is convex, but it has to be minimized under constraints. To solve this minimization problem, we propose to resort to a parallel forward-backward based Primal-Dual approach Condat (2013); Vũ (2013) (see also Komodakis and Pesquet (2014) for a tutorial about these methods). We end up with Algorithm 2 in Appendix C This approach 
presents the advantage of a reduced complexity per iteration as no linear operator inversion is required, while ensuring convergence guarantees.

Starting from the resulting regularized TV-based solution $d^{*}$, we apply morphological operations in order to detect the voxels containing structures suspected to be microcalcifications whose size lies in a specific range, in the line of the method from Wheeler et al. (2006). As we are interested in preserving small particles with no specific spatial orientation, we use an opening operator using a disk with a small radius as the structuring element that we apply slice per slice. In order to keep structures with radius size in the range $\left[\rho_{\min }, \rho_{\max }\right]$ where $0<\rho_{\min }<\rho_{\max }$, we apply the following steps:

1. Apply opening with radius $\rho_{\min }$ on $d^{*}$ to generate $d_{\rho_{\min }}^{*}$.

2. Apply an opening with radius $\rho_{\max }$ on $d_{\mid \rho_{\min }}^{*}$ to generate $d_{\mid \rho_{\max }}^{*}$.

3. $\mu=d_{\mid \rho_{\min }}^{*}-d_{\mid \rho_{\max }}^{*}$.

The output of the above nonlinear method leads to our estimated $\mu$ which is expected to contain high intensity voxels in zones where structures are present in the predefined radius range, and very low intensity ones otherwise.

\subsubsection{Construction of the ROIs}

The estimated signal $\mu$ will also be useful for the construction of the ROIs themselves, as the local maxima of $\mu$ correspond to sought areas for microcalcifications. We propose to extract local maxima from a MaxTree Berger et al. (2007) representation computed using 26-connectivity. This approach allows to represent the volume as a tree where each node corresponds to a connected component of similar graylevel. The nodes are then connected hierarchically depending on their intensity. More precisely, a parent of a tree node corresponds to a neighboring pixel having a given intensity $I^{\prime}$ lower than the pixel value $I$ of its child. By construction, the leaves represent nodes which have not be identifed as parents, and they have locally the highest graylevel intensities, Determining the set of pixels corresponding to each leaf allows us to construct a list of $q$ representative pixels that we define to be the centers of our ROIs. We then deduce the $\left(\mathbb{I}^{i}\right)_{1 \leq i \leq q}$ sets by imposing a minimum ROI size enabling the inversion of the covariance matrix, while minimizing the overlap of ROIs.

\subsubsection{Estimation of the channelized noise covariance matrix}

The estimation of $\Sigma$ has been performed independently of the estimation of $\mu$ on a training set of ROIs. The procedure is described in Appendix B. As mentioned before the channel mechanism enables a simpler computation of the inverse covariance matrix compared to HO by greatly reducing the dimension of the training regions of interest Myers and Barrett(1987) and requires fewer training images than $\mathrm{HO}$ Xin and Park (2013).

\section{Spatially Adaptive TV-based regularization}

In literature, most DBT reconstructions have used total variation (TV) as a spatial regularization function Sidky et al. (2007. 2009); Metin et al. (2014). The TV regularizer has demonstrated great performance in several applications of image processing Condat (2017); Chambolle et al. (2011); Abergel and Moisan (2017); Corbineau et al. (2018). However, the staircasing effect inherent to TV Nikolova (2000) is not desirable as it rarely fits with the natural textures and shapes arising in real images. Several research studies have suggested alternative TV-based penalties, by proposing smooth approximation of it Aujol (2009); Blomgren et al. (1997); Chan et al. (2000, 2007), by introducing non-local similarity measures Chierchia et al. (2014), by considering the posterior mean estimate instead of the standard maximum a posteriori one Louchet and Moisan (2008), or by investigating anisotropic gradient operators Luo et al. (2018); Merveille et al. (2016); Liao and Shao (2017); Xu et al. (2018); Biton and Gilboa (2018). 
The aforementioned works gave rise to promising results, but may however not be adapted to the restoration of very heterogeneous areas, such as the breast volume imaged in DBT. We thus propose to formulate a new Spatially Adaptive TV (SATV) regularization function which will take into consideration the different morphological contents of the breast. Different regularizers are applied, depending if microcalcifications are expected or not in the considered image zones. To this aim, we introduce a new definition of the gradient field allowing to involve heterogeneous prior knowledge on the structural content of each voxel of the sought volume.

\subsection{Mathematical formulation}

Let us consider the signal map $\mu \in \mathbb{R}^{m}$ obtained as an output of the construction of the previously introduced detectability regularization function (8), which is normalized between 0 and 1 . This map is of great interest, as it provides a good estimate for the location of the potential microcalcifications in the volume. This allows us to define the following weight parameters,

$$
(\forall i \in\{1, \ldots, m\}) \quad \lambda_{i}= \begin{cases}1 & \text { if } \mu_{i} \leq v-\theta \\ \frac{\theta+v-\mu_{i}}{2 \theta} & \text { if } v-\theta \leq \mu_{i} \leq v+\theta \\ 0 & \text { otherwise, }\end{cases}
$$

with $v \in[0,+\infty[$ and $\theta \in] 0, \min (v, 1-v)]$ some threshold values which allow to transition between the breast texture and the detected microcalcifications, and that will be set so as to preserve the geometric shape of the detected microcalcifications. By construction, $\left(\lambda_{i}\right)_{1 \leq i \leq m} \in[0,1]^{m}$. The latter weight vector will allow us to address differently the regularization of the plain healthy tissue and of the critical zones potentially containing microcalcification objects.

For this purpose, we formulate our SATV regularization function as follows,

$$
\left(\forall d \in \mathbb{R}^{m}\right) \quad \Phi(d)=\sum_{i=1}^{m} \psi\left((1-\eta) \lambda_{i} \Delta_{i} d+\eta\left(1-\lambda_{i}\right) \delta d_{i}\right),
$$

where $\delta=\left[\begin{array}{lll}1 & 1 & 1\end{array}\right]^{\top}, \psi: \mathbb{R}^{3} \rightarrow \mathbb{R}$ represents a sparsity promoting function which applies either on the image intensity or on its gradients, $\eta \in[0,1]$ controls the overall contribution of each regularizing term, and $\Delta_{i}$ denotes the $3 \mathrm{D}$ gradient operator at voxel $i \in\{1, \ldots, m\}$, that is expressed as

$$
\left(\Delta_{i}\right)^{\top}=\left[\begin{array}{lll}
\Delta_{i}^{x} & \Delta_{i}^{y} & \Delta_{i}^{z}
\end{array}\right]^{\top}
$$

with $\Delta_{i}^{x}$ (resp. $\left(\Delta_{i}^{y}\right)$ and $\left.\left(\Delta_{i}^{z}\right)\right)$ discrete horizontal, (resp. vertical and depth) gradient operators assuming zero-boundaries. It is worthy to point out that if $\lambda_{i}=0$ for every $i \in\{1, \ldots, m\}$, and $\psi=\|\cdot\|_{1}$, then $\Phi$ corresponds to the $\ell_{1}$ regularization, while if $\lambda_{i}=1$ for every $i \in\{1, \ldots, m\}$, and $\psi=\|\cdot\|$, then $\Phi$ reduces to the standard isotropic TV regularizer. For a suitable weight vector $\left(\lambda_{i}\right)_{1 \leq i \leq m}$, the norm of the gradient of the image will be penalized in regions where no microcalcifications are expected, while the intensity of the microcalcifications will be penalized in the complementary regions.

Equivalently, we can reformulate (13) as,

$$
\left(\forall d \in \mathbb{R}^{m}\right) \quad \Phi(d)=\Psi(L d)
$$

with

$$
\left(\forall u=\left(u_{i}\right)_{1 \leq i \leq m} \in\left(\mathbb{R}^{3}\right)^{m}\right) \quad \Psi(u)=\sum_{i=1}^{m} \psi\left(u_{i}\right),
$$

and

$$
L=(1-\eta)\left(\Lambda \otimes I_{3}\right) \Delta+\eta\left(I_{m}-\Lambda\right) \otimes \delta \in \mathbb{R}^{(3 m) \times m},
$$

where $\otimes$ denotes the Kronecker product, $I_{m}$ states for the identity matrix of dimension $m \times m$ and

$$
\Lambda=\operatorname{Diag}\left(\lambda_{1}, \ldots, \lambda_{m}\right),
$$


where $\operatorname{Diag}(\cdot)$ is the diagonal matrix with the elements of its vector argument on the main diagonal.

Different strategies can be adopted for the choice of the sparsity promoting function $\psi$, which will have an impact on the reconstruction algorithm used to solve Problem (3)-(4). For instance, if $\psi$ is convex, and its proximity operator has a closed form expression (e.g., $\psi$ is the $\ell_{1}$ or $\ell_{2}$ norm), proximal algorithms Combettes and Pesquet (2011.2021) which include ADMM Boyd et al. (2011) and primal-dual methods Komodakis and Pesquet (2014) can be used. If $\psi$ is Lipschitz-differentiable, but not necessarily convex (e.g. smooth approximations of $\ell_{1}$ or $\ell_{0}$ ), then the problem can be solved using majoration-minimization (MM) schemes Sun et al. (2017); Jacobson and Fessler (2007), which have shown their efficiency in the context of image tomography Chouzenoux et al. (2013ba). In this work, we will adopt the latter approach, and we will set

$$
\left(\forall u \in \mathbb{R}^{3}\right) \quad \psi(u)=\beta \sqrt{\|u\|_{2}^{2}+\epsilon^{2}},
$$

where $\beta>0$ is the regularization weight, and $\epsilon>0$ controls the smoothness of the penalty function. Since all the involved terms of Problem (3)-(4) are now differentiable, we will resort to an MM algorithm tailored for such smooth function, that will be detailed in the next section.

\section{Majorize-minimize memory gradient algorithm for DBT reconstruction}

The DBT reconstruction problem amounts to minimizing the objective function:

$$
\left(\forall d \in \mathbb{R}^{m}\right) \quad f(d)=\frac{1}{2}\|p-A d\|_{2}^{2}-\alpha D(d)
$$

$$
+\Psi(L d)+\frac{\gamma}{2}\|d\|_{2}^{2}+\kappa Q(d)
$$

MM algorithms rely on successive majorizing approximations of function $f$ in order to produce a sequence of iterates that will converge to a solution to the problem, under suitable assumptions. The MM framework gives rise to a class of efficient and flexible optimization algorithms that is grounded on solid theoretical foundations. MM algorithms are very popular in the field of medical image reconstruction De Pierro (1995); Erdogan and Fessler (1999); Florescu et al. (2014). The Majorize-Minimize Memory Gradient (3MG) algorithm falls within this class. Integrating a subspace acceleration strategy in the standard quadratic MM algorithm indeed constitutes one of the most efficient strategies for smooth optimization at large scales Chouzenoux and Pesquet (2016) and one of the few benefiting from convergence guarantees in the non-convex case Chouzenoux and Pesquet (2017); Chouzenoux et al. (2013a). In this section, we make explicit the formulation of 3MG algorithm when applied to Problem (3)-(4) (see detailed derivations in Appendix D Furthermore, we propose two numerical improvements of the algorithm, which aim to further improve the speed of the reconstruction process.

\subsection{Majorize-Minimize Memory Gradient method}

In order to implement the $3 \mathrm{MG}$ approach Chouzenoux et al. (2013a) to minimize [20, we must construct a quadratic tangent majorant function for $f$ at $d^{\prime}$, taking the form:

$$
\left(\forall d \in \mathbb{R}^{m}\right) \quad q\left(d, d^{\prime}\right)=f\left(d^{\prime}\right)+\nabla f\left(d^{\prime}\right)^{\top}\left(d-d^{\prime}\right)
$$

$$
+\frac{1}{2}\left(d-d^{\prime}\right)^{\top} \mathcal{M}\left(d^{\prime}\right)\left(d-d^{\prime}\right)
$$

where $\mathcal{M}\left(d^{\prime}\right) \in \mathbb{R}^{m \times m}$ denotes a symmetric positive semi-definite matrix that ensures Properties $(\mathrm{D} .3)$. Using the same procedure than in Chouzenoux et al. (2013a), we can show that this matrix can be defined as

$$
\left(\forall d \in \mathbb{R}^{m}\right) \quad \mathcal{M}(d)=A^{\top} A+L^{\top} \operatorname{Diag}(b(d) \otimes \delta) L+(\gamma+2 \kappa) I_{m} .
$$


Hereabove, $b(d)=\left(b_{i}(d)\right)_{1 \leq i \leq m} \in \mathbb{R}^{m}$ is such that, for every $i \in\{1, \ldots, m\}$,

$$
b_{i}(d)=\beta\left(\left\|(L d)_{i}\right\|_{2}^{2}+\epsilon^{2}\right)^{-1 / 2}
$$

where $(L d)_{i}$ is the $i$-th block of 3 components of $L d$. The construction of the above majorant approximation yields Algorithm 1 summarizing the $3 \mathrm{MG}$ iterations applied to the minimimization of [20], where $\dagger$ denotes the pseudo-inverse operation. It is worth noticing that the latter operation has a very limited complexity in our case, as it reduces to the inversion of a $2 \times 2$ matrix. The reader can found the detailed rational behind each step of the $3 \mathrm{MG}$ algorithm in Appendix D. In practice, we initialize the $3 \mathrm{MG}$ algorithm by using $d^{(0)}$ as the Filtered Backprojection (FBP) reconstruction and we implement a maximum iteration number $J_{\max }$.

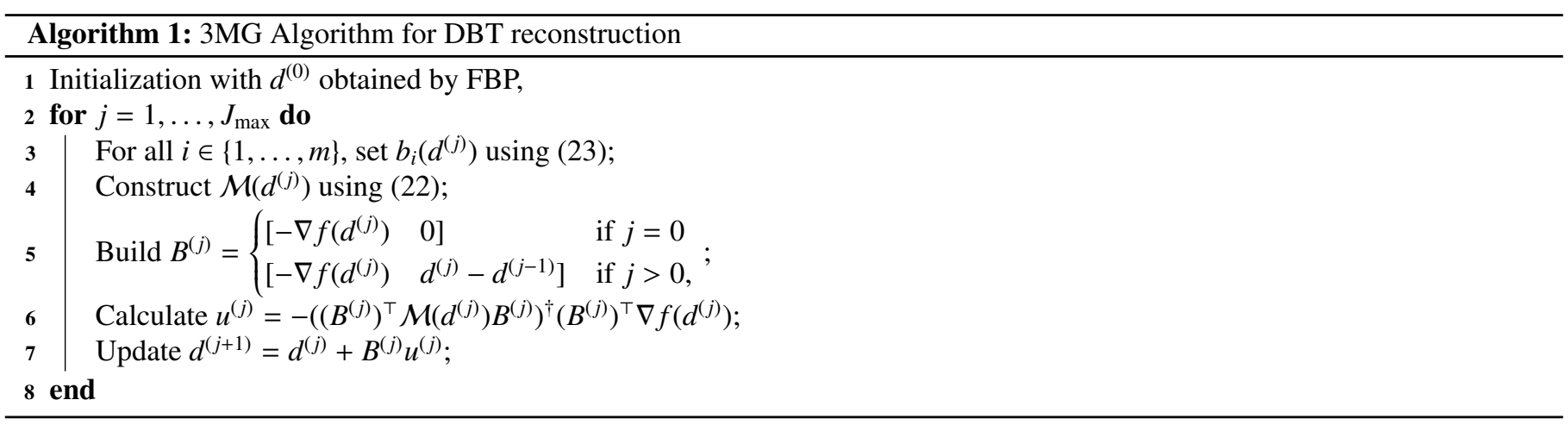

\subsection{Practical implementation}

Function $Q$ defined in (5) penalizes the distance of each component of $d$ to the hypercube $\left[0, d_{\max }\right]^{m}$. The strength of this penalization is controlled through the weight $\kappa$. The greater $\kappa$, the closer to the indicator function of the constrained domain $\kappa Q$ is, hence the more faithfully the constraints are fulfilled. However, increasing $\kappa$ also induces an increase of the curvature of the majorizing function $q$, leading to a slow down of the convergence of the $3 \mathrm{MG}$ algorithm, in practice. To address this issue, we propose two modifications to the algorithm.

We first substitute 22, by

$\left(\forall d \in \mathbb{R}^{m}\right) \quad \widetilde{\mathcal{M}}(d)=A^{\top} A$

$$
+L^{\top} \operatorname{Diag}\{b(d) \otimes \delta\} L+\gamma I_{m}+\operatorname{Diag}\{s(d)\}
$$

where, for every $i \in\{1, \ldots, m\}$, the $i$-th component of vector $s(d)$ is

$$
s_{i}(d)= \begin{cases}0 & \text { if } d_{i} \in\left[0, d_{\max }\right] \\ 2 \kappa & \text { otherwise }\end{cases}
$$

This amounts to relaxing the majorizing assumption (D.3) by considering only a local majoration for $f$ in the neighborhood of the current iterate.

Second, in order to further mitigate the negative impact of a large value of $\kappa$ on the convergence speed, we consider to progressively increase this weight along iterations according to the following rule

$$
(\forall j \in \mathbb{N}) \quad \kappa^{(j)}=\kappa_{\max } \frac{j}{j+\xi}
$$

where $\kappa_{\max }$ is a maximum value for $\left(\kappa^{(j)}\right)_{j \in \mathbb{N}}$ and $\xi \geq 0$ is a parameter controlling the evolution of this sequence along iterations. When $j$ goes to infinity, $\kappa^{(j)} \rightarrow \kappa_{\max }$. For small values of $\xi$, a large penalization weight is obtained from the very beginning of 
the algorithm whereas, large values of $\xi$ yields a more gradual penalization over the range of the pixel intensities. When $\xi=0$, a constant parameter $\kappa^{(j)} \equiv \kappa_{\max }$ is retrieved.

\section{Applicative results}

We now present results to assess the performance of our proposed method on physical phantoms and clinical datasets, that will be further detailed in Sections 6.3.1 and 6.3.2

\subsection{Methodology}

For each considered volume, we simulate the 9 projections obtained using a DBT commercial system (Senographe Essential, GE Healthcare) with an angular range of $25^{\circ}$. The detector is composed of $3062 \times 2394$ detector elements of $100 \mu \mathrm{m}^{2}$ size. The volumes are reconstructed on a $100 \mu \mathrm{m} \times 100 \mu \mathrm{m} \times 1 \mathrm{~mm}$ sampling grid. We aim at enhancing the detectability of microcalcifications with size ranging from $0.2 \mathrm{~mm}$ to $0.4 \mathrm{~mm}$. This amounts to focusing on a specific group of microcalcification surrogates included in the physical accreditation phantom Model 015 (CIRS). The detection map $\mu$ is generated as explained in Section 3.2.1 using the aforementioned size range. The size of the ROIs is $51 \times 51 \times 5$ pixels. The total number $q$ of ROIs depends on each considered dataset. In our experiments, $q$ varies from $10^{5}$ to $10^{6}$. Then we derive the volume $\left(\lambda_{i}\right)_{1 \leq i \leq m}$ as explained in Section 4.1 We set up experimentally the threshold $v$ in $(12)$ so that the geometric shape of the detected microcalcifications is preserved. The parameter $\theta$ is manually finetuned so as to preserve an optimized transition between breast background and microcalcifications. We now describe the learning process that we use to set the covariance matrix $\Sigma$. We consider a database of 400 ROIs generated by using a simulation software developed by GE Healthcare Li et al. (2018b). The ROIs contain a predefined type of background (e.g., uniform, textured). Furthermore, half of the ROIs contain lesions of $0.2 \mathrm{~mm}$ in size, while the others do not contain any. LaguerreGauss channels Park et al. (2007) are employed to define matrix $U=\left[\begin{array}{llll}u_{1} & u_{2} & \ldots & u_{c}\end{array}\right] \in \mathbb{R}^{k \times c}$ where, for every $p \in\{1, \ldots, c\}$, the $p$-th column of the matrix, $u_{p}=\left(u_{\ell, p}\right)_{1 \leq \ell \leq k}$ represents a $k$-dimensional Laguerre-Gauss channel whose components are given by

$$
(\forall \ell \in\{1, \ldots, k\}) \quad u_{\ell, p}=
$$

$$
\frac{\sqrt{2}}{a_{u}} \exp \left(\frac{-2 \pi\left\|z_{\ell}\right\|_{2}^{2}}{a_{u}^{2}}\right) L_{p-1}\left(\frac{2 \pi\left\|z_{\ell}\right\|_{2}^{2}}{a_{u}^{2}}\right)
$$

with $z_{\ell}$ the $3 \mathrm{D}$ spatial coordinates of the $\ell$-th voxel of the ROI (with spatial origin defined at the center of the ROI), $a_{u} \in \mathbb{R}_{+}$the spread of the Gaussian kernel, and $L_{p-1}$ the Laguerre polynomial of degree $p-1$, defined as

$$
(\forall x \in \mathbb{R}) \quad L_{p-1}(x)=\sum_{j=0}^{p-1}(-1)^{j}\left(\begin{array}{c}
p-1 \\
j
\end{array}\right) \frac{x^{j}}{j !} .
$$

Athough the employed functions have a spherical symmetry and microcalcifications might be non-isotropic, the use of anistropic functions would lead to more complex forms of $U$. A range of values for $a_{u}$ and $c$ are considered depending on the background type. More precisely, we selected the optimal combination maximizing the area under ROC curve, by following the CHO framework Platiša et al. (2011). Values of $\left(a_{u}, c\right)$ equal to $(0.8,30)$ and $(0.6,30)$ are used, for uniform and textured background respectively, following previous research works $\mathrm{Li}$ et al. (2018b). The parameter $\zeta$ in $10 \mathrm{~b}$ is chosen equal to $\frac{3}{2}\left\|\Delta d_{0}\right\|_{1,2}$, leading to satisfying visual results where $d_{0}$ is taken as the FBP solution. Finally, the SATV parameters are adjusted for each dataset, as further detailed in Sections 6.3.1 and 6.3.2 


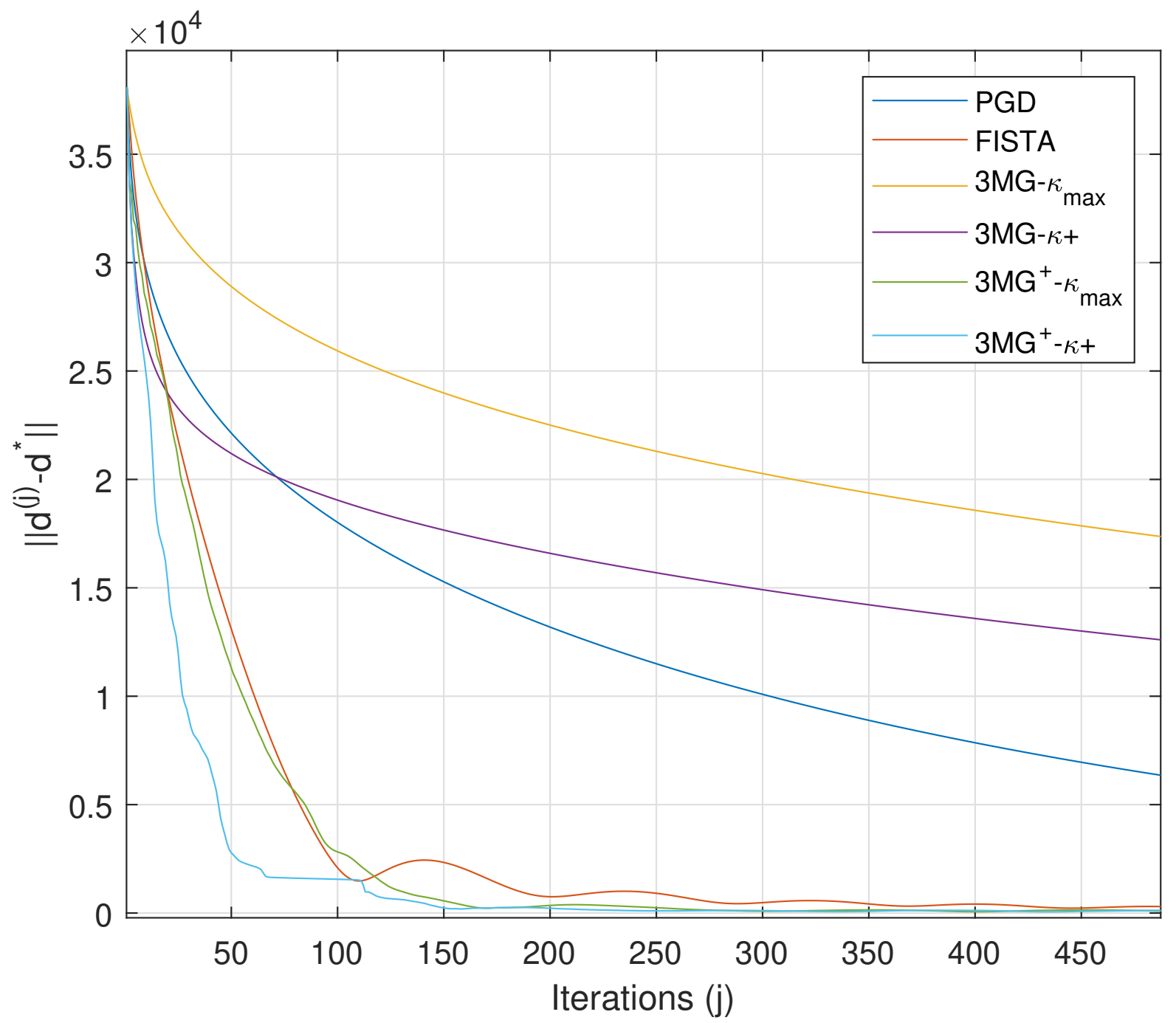

Fig. 2: Convergence rate comparison between PGD, FISTA and 3MG with its variants on clinical data in terms of $\left\|d^{(j)}-d^{*}\right\|$ where $d^{*}$ is the reconstructed volume for each algorithm at iteration $10^{4}$.

\subsection{Numerical performance of $3 M G$ algorithm}

We illustrate the practical convergence profile of the proposed reconstruction algorithm on a BI-RADS d clinical dataset. Note that class d corresponds to extremely dense breasts American College of Radiology et al. (2013). We solve the optimization Problem (3)-(4) with 3MG method, either using its initial form described in Algorithm 1, or its variants proposed in Section 5.2 We also perform comparisons with two state-of-the-art optimization methods, namely FISTA Chambolle and Dossal (2015) and the projected gradient descent (PGD) Calamai and Moré (1987), where we imposed the range constraint $d \in\left[0, d_{\max }\right]^{m}$. All the algorithms have been initialized with FBP solution and ran until $10^{4}$ iterations. We set the elastic net weight $\gamma=1$ for all the experiments, which allows to ensure the uniqueness of the solution $d^{*}$ while not degrading the overall image quality of DBT. We refer to Algorithm 1 with modified majorant 240 as $3 \mathrm{MG}^{+}$. Moreover, we distinguish the cases when $\kappa^{(j)} \equiv \kappa_{\max }$, designated by $3 \mathrm{MG}-\kappa_{\max }$ or $3 \mathrm{MG}^{+}-\kappa_{\max }$, from the cases when the varying rule 26 is adopted, designated by $3 \mathrm{MG}-\kappa^{+}$and $3 \mathrm{MG}^{+}-\kappa^{+}$. We set $\kappa_{\max }=10^{3}$, since it was observed to lead to the same image quality at convergence as when using FISTA and PGD algorithms. Furthermore, we set $\xi=75$ in 26 since it was observed to achieve the best trade-off between convergence speed and image 
quality. Figure 2 illustrates the evolution of the distance to the solution $d^{*}$, computed after a large number of iterations equal to $10^{4}$. We notice that $3 \mathrm{MG}-\kappa_{\max }$, that corresponds to the standard 3MG implementation, leads to the slowest convergence, while 3MG+$\kappa_{\max }$ reaches similar performance to FISTA. Finally, $3 \mathrm{MG}^{+}-\kappa+$ outperforms the other competitors in terms of convergence speed. These results allow to assess that both proposed procedures in Section 5.2, when combined together, do significantly improve the convergence speed of 3MG, yielding a reconstruction method that outperforms all its competitors in this example.

\subsection{Qualitative assessment of the proposed DBT reconstruction approach}

We now illustrate the visual quality of the results obtained by using the proposed DBT reconstruction approach. In the remainder of this section, we will make use of $3 \mathrm{MG}^{+}-\kappa^{+}$, as a minimization algorithm, for the resolution of Problem (3)-(4). The algorithm is initialized with FBP solution and run until a maximum number of iterations (here equal to 200), which is sufficient to ensure a practical convergence in our experiments. For all of them, we set the weight of the elastic net $\gamma=1$.

\subsubsection{Physical phantom data}

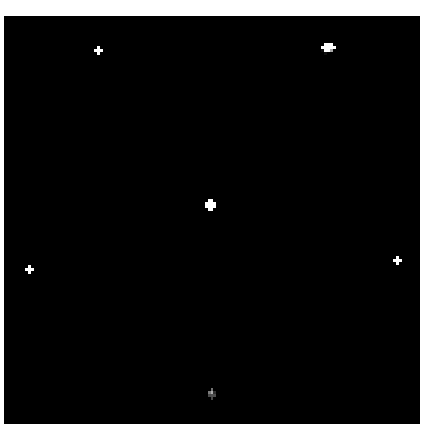

(a)

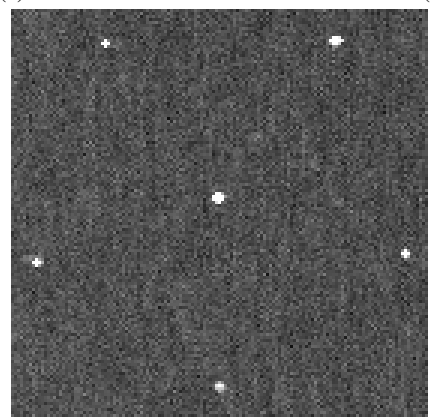

(e)

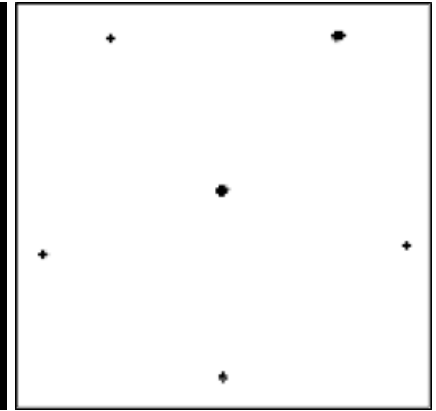

(b)

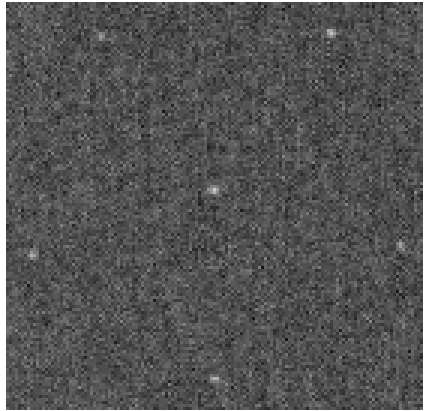

(c)

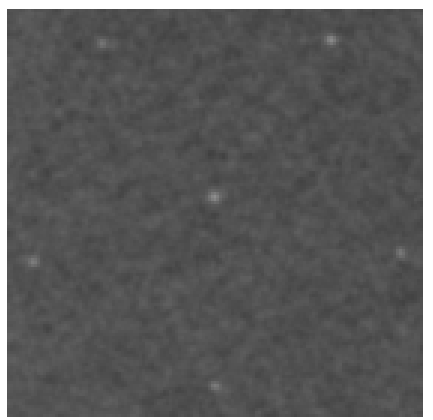

(d)

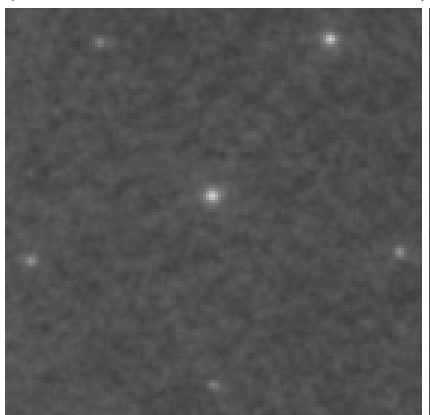

(f)

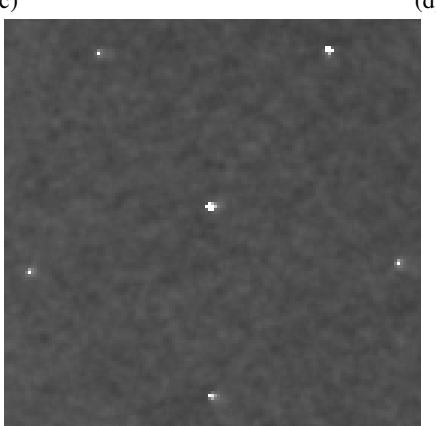

(g)

Fig. 3: Region of ACR phantom slice containing 6 ROIs : (a) Detection map $\mu$. (b) Weights $\left(\lambda_{i}\right)_{1 \leq i \leq m}$. (c) Non regularized least squares solution (NRLS). (d) DBT reconstruction with classical TV regularization and no detectability function (TV). (e) NRLS with detectability function (dNRLS). (f) DBT reconstruction with and classical TV regularization (dTV). (g) DBT reconstruction with detectability function and SATV regularization (dSATV).

We first assessed our method when using the physical accreditation phantom Model 015 (CIRS) with dimension $45 \mathrm{~mm} \times$ $102 \mathrm{~mm} \times 108 \mathrm{~mm}$. For the sake of illustration, we only display a zoomed region containing $6 \mathrm{Al}_{2} \mathrm{O}_{3}$ specks simulating microcalcifications of size $0.32 \mathrm{~mm}$. We first show in Fig. 3 a) the detection map derived from our method presented in Section 3.2.1 Note that the background is merely equal to 0 and that all the 6 microcalcifications have been detected. Following our SATV approach, for each microcalcification intensity, a different regularization level is applied. The obtained values for $\left(\lambda_{i}\right)_{1 \leq i \leq m}$ described in Section 4.1 are shown in Fig. 3 (b). We set $v=1.5 \times 10^{-3}, \theta=v / 3$, and $\alpha=750$.

As expected, the non regularized least squares solution (NRLS) leads to a highly noisy DBT reconstruction (Fig. 3 ( c)). DBT reconstruction with classical TV and no detectability function (TV) leads to smoother background but reduces microcalcification 
intensity and sharpness (Fig. 3 d d)). When the detectability function is used, without additional regularization (dNRLS), we enhance and sharpen microcalcifications but a noisy background is still present (Fig. 3.e)). By combining the detectability function and the classic TV regularization (dTV), (Fig. 3ff)), we both reduce the noise in the background and enhance microcalcification visibility. However, the enhanced microcalcifications appear less sharp and with a slight spreading effect, when compared to Fig. 3.e). Therein lies the interest of our novel SATV regularization. SATV regularization combined with the detectability function (dSATV) significantly enhances the visibility as well as the sharpness of the detected microcalcifications, while ensuring a proper denoising of the background (Fig. $3 \mathrm{G}$ )). This comparison between the SATV-based reconstruction and a classical TV-based reconstruction was conducted with regularization parameters, namely $\beta$ and $\eta$, set to restore the background with the same level of quality in both approaches. In this example, $\beta=600, \epsilon=10$ for classical TV while $\beta=630, \epsilon=10$ and $\eta=0.048$ in the case of the new SATV regularization.

\subsubsection{Clinical data}

We have also evaluated our proposed approach on clinical images. In Figs. 4 and 5 we present results for two cases presenting a BI-RADS d breast composition (the breasts are extremely dense) containing an isolated microcalcification and a cluster of microcalcifications respectively. In Fig. 6, we show a case presenting a BI-RADS c breast composition (the breasts are heterogeneously dense) comprising a potentially malignant cluster of microcalcifications. The corresponding detection maps are shown in Fig. 4(a), Fig. 5.a) and Fig. 65a), respectively. The constructed maps for $\left(\lambda_{i}\right)_{1 \leq i \leq m}$ obtained following the approach described in Section 4.1 are shown in Fig. 4(b), Fig. 5(b) and Fig. 6(b). Regarding the hyper-parameters, we used $\beta=600$ and $\epsilon=10$, for classical TV and $\beta=660, \eta=0.09$, and $\epsilon=10$ when SATV regularization is applied. Furthermore, we set $\theta=v / 3$, with $(v, \alpha)=(0.09,1500)$ for the first example, $(v, \alpha)=(0.03,2500)$ for the second volume and $(v, \alpha)=(0.009,500)$ for the third one.

Similar conclusions as for the physical phantom case, can be drawn from visual inspection of those images. We notice again two drawbacks of DBT reconstructions using a detectability function and no TV regularization. First, it may yield saturated microcalcifications since no penalization is applied on their gray level values (Fig. 4 (e)). Second, some false positives in the detection map may also lead to enhanced microcalcification-like structures when no regularization is applied (Fig. 5(e) and Fig. 6(e)). By combining the detectability function and TV regularization (Fig. 4ff), Fig. 5(f) and Fig.6ff), we observe similar results as for the physical phantom case, enhanced but unsharp microcaclifications and a denoised background. Hereagain, we highlight the advantage of our proposed approach (Fig.4 $4 \mathrm{~g}$ ), Fig. 5.g) and Fig.6.g)) that simultaneously enables a robust preservation of the enhanced microcalcification shape and a high quality restoration of the background. It is worth mentioning that the reconstruction with SATV and classical TV can provide the same breast texture quality, after a suitable choice for the regularization parameters $\eta$ and $\beta$.

\subsection{Quantitative assessment of the proposed DBT reconstruction approach}

We finally perform a quantitative evaluation of our proposed dSATV reconstruction approach on clinical data. To this aim, we conduct a visual experiment trial where experts compare dSATV and NRLS reconstructions, regarding several aspects including microcalcification conspicuity, rendering of breast structures, presence of potential artifacts and overall visual preference. The methodology and the results of our experiment are presented hereafter.

\subsubsection{Image data set}

We have extracted 19 regions of interest from 16 clinical cases presenting BI-RADS $\mathrm{c}$ and $\mathrm{d}$ breast compositions, acquired with the same DBT system (Senographe Essential, GE Healthcare). Each of the 19 trial image was $220 \times 220 \times 7$ voxels in size, four of them contain isolated microcalcifications while the remaining ones display various types of microcalcification groups. We then 


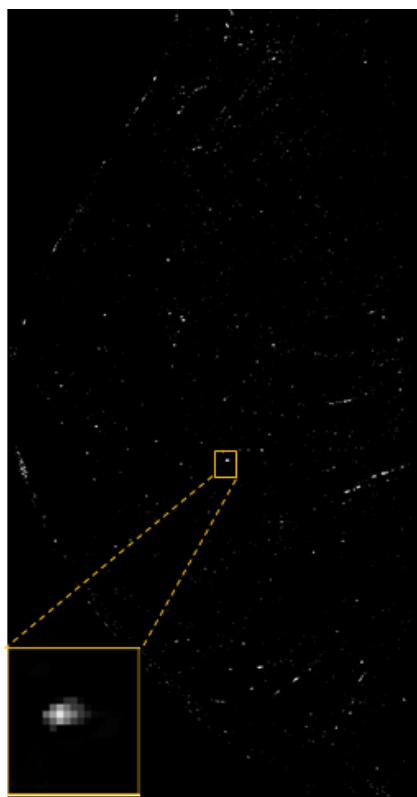

(a)

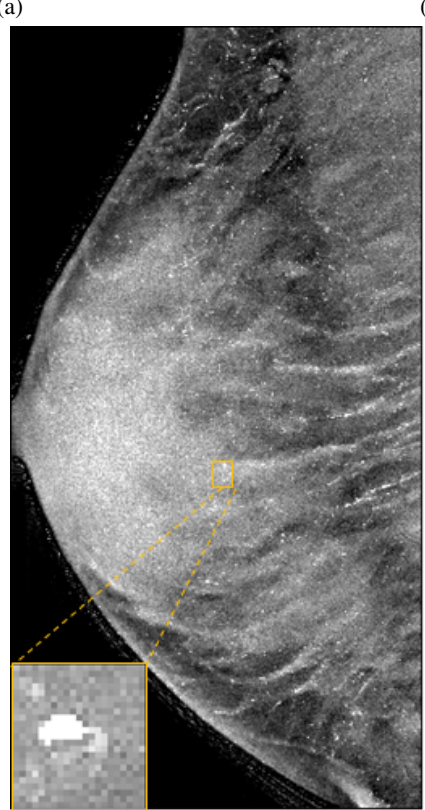

(e)

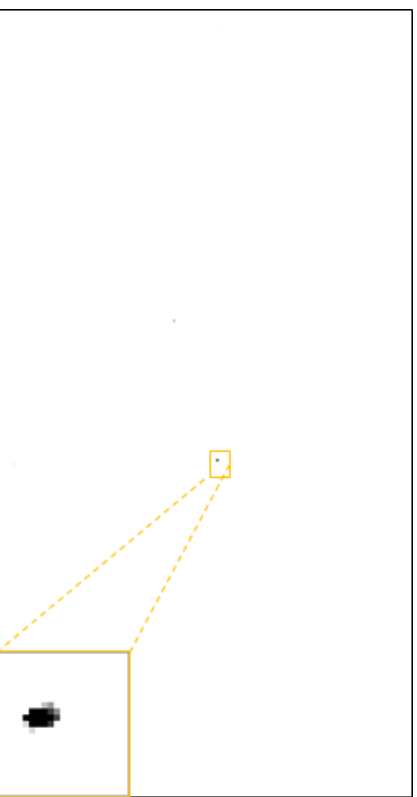

(b)

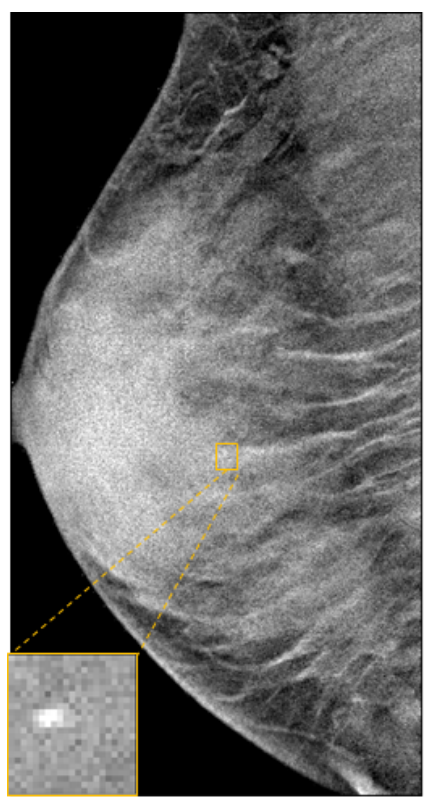

(c)

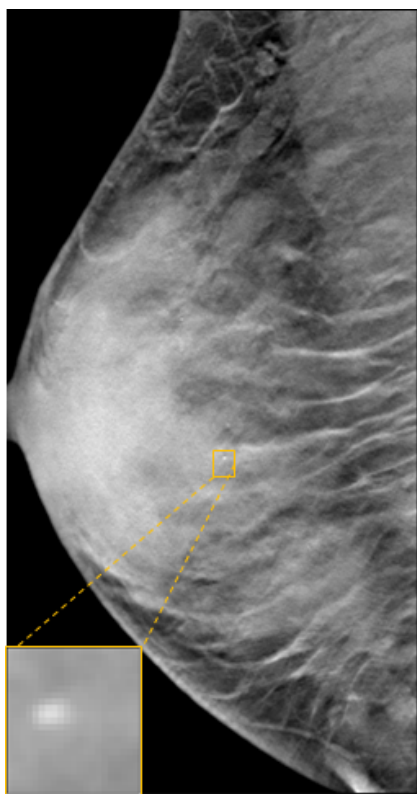

(d)

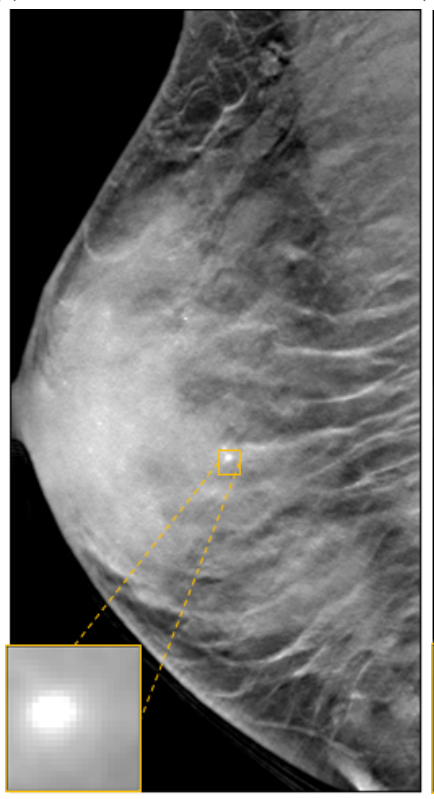

(f)

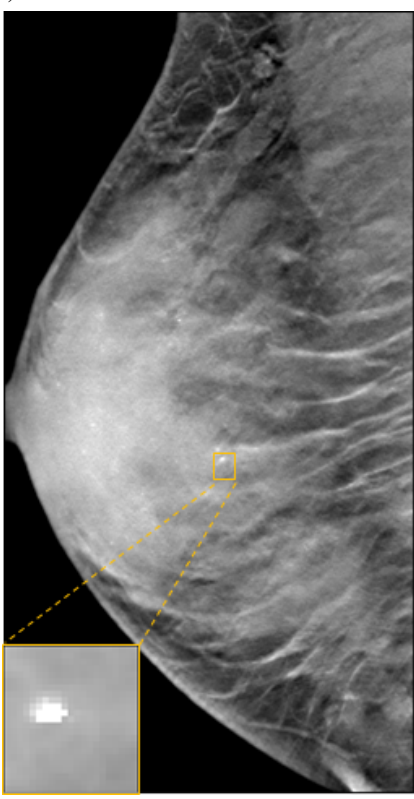

(g)

Fig. 4: Selected slice of a DBT reconstruction with size $2344 \times 868 \times 44$ (voxels) (a) Detection map $\mu$. (b) Weights $\left(\lambda_{i}\right)_{1 \leq i \leq m}$. (c) NRLS. (d) TV. (e) dNRLS. (f) dTV. (g) dSATV.

reconstructed each case with both the NRLS solution and our proposed dSATV solution. For the latter, the parameters were set following the same approach as detailed in Sections 6.2 and 6.3 . For each test case, we built visual trials displaying side by side (with random left/right side assignation) both reconstruction results.

\subsubsection{Image review}

Fourteen qualified participants, namely nine radiologists and five GE Healthcare image quality experts in mammography, participated in our visual experiment. No training session was conducted before the actual experiment. Each reader saw sequentially, in the same order, the 19 trial image pairs. The 7 slices of each image pair were displayed using cine-loop review mode. Between two consecutive trials, a uniform neutral gray image was displayed. For each trial, the reader was asked to choose between right or left image (see slice examples in Figure 7 ) by answering the following questions : 

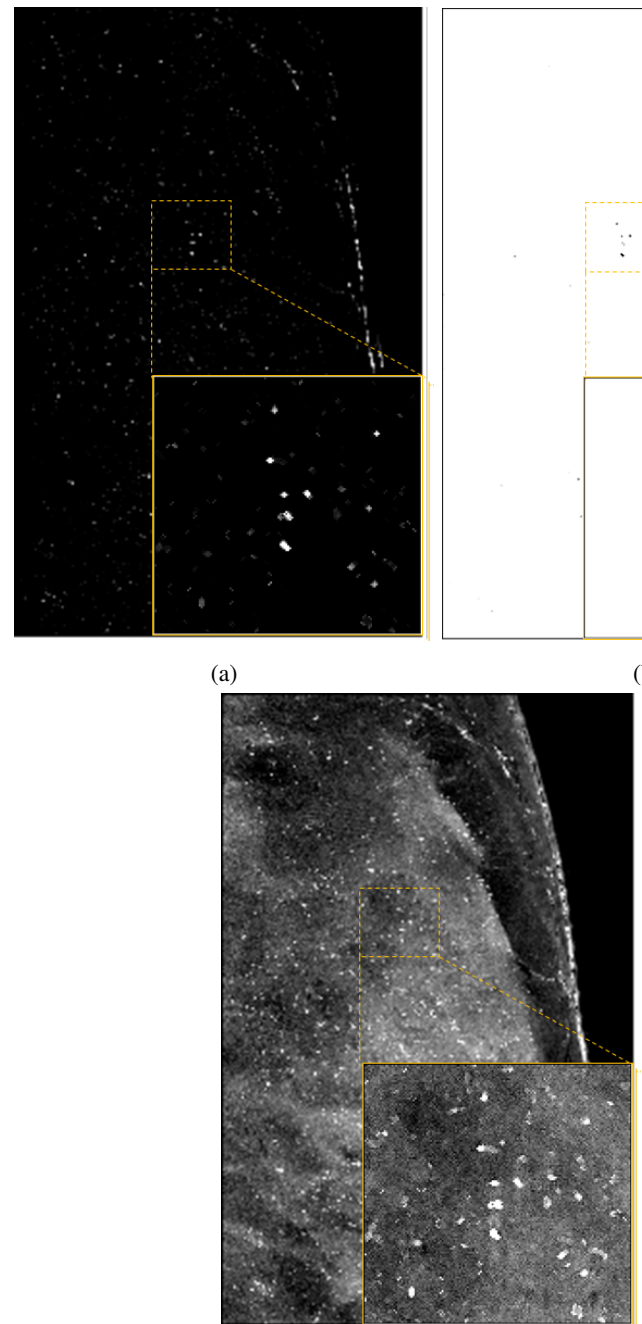

(e)

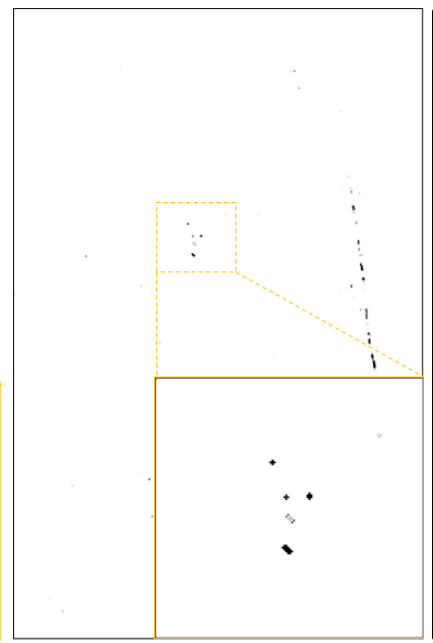

(b)

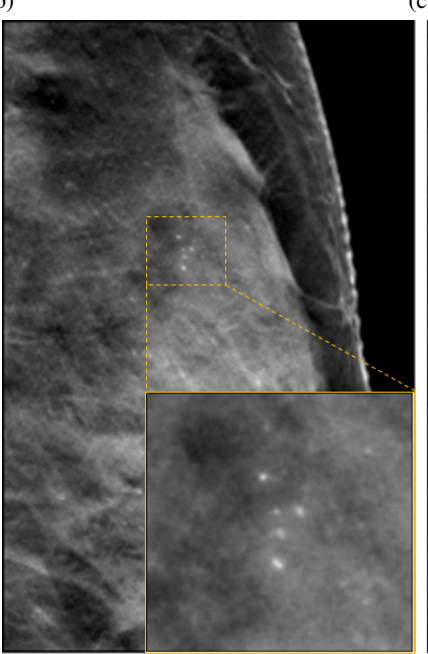

(f)
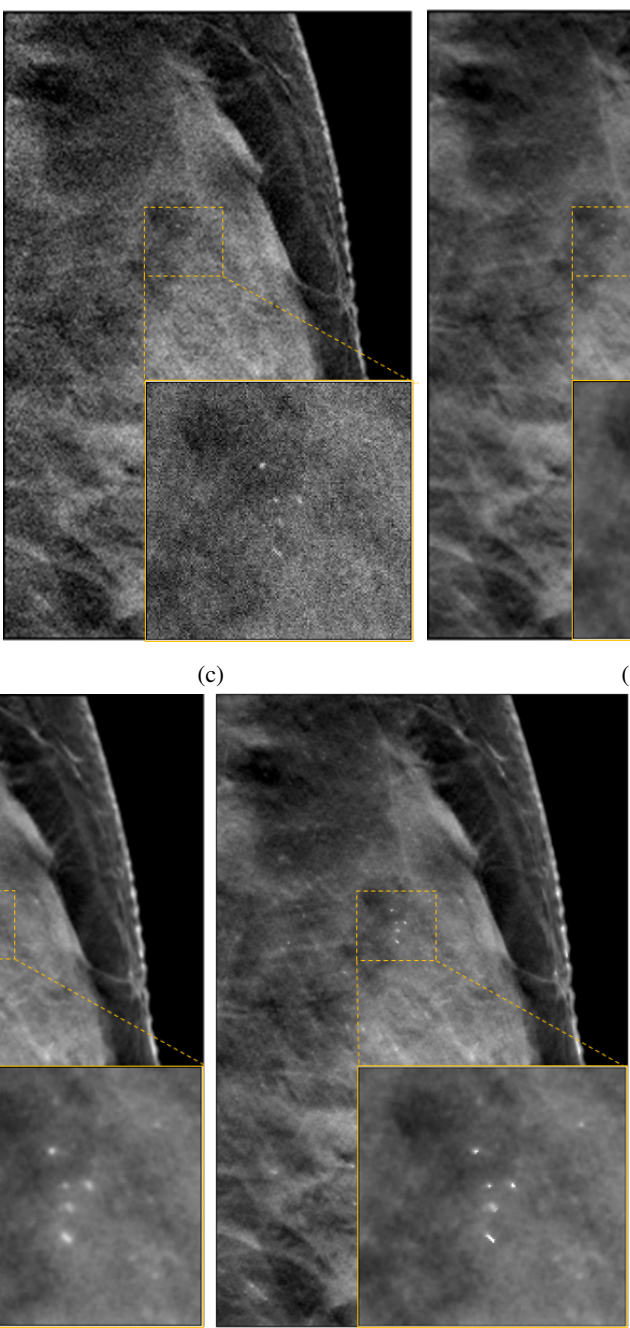

(g)

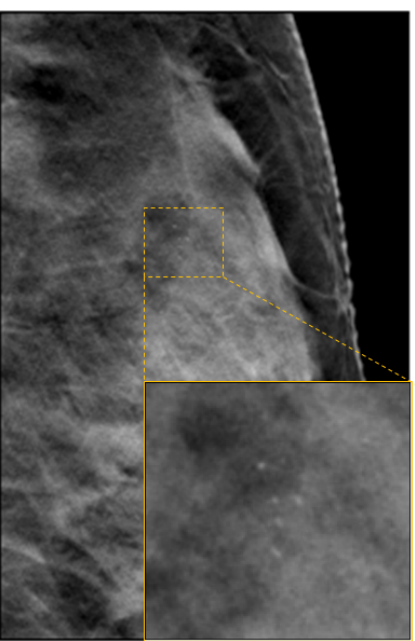

Fig. 5: Selected slice of a DBT reconstruction with size $2227 \times 594 \times 44$ (voxels) (a) Detection map $\mu$. (b) Weights $\left(\lambda_{i}\right)_{1 \leq i \leq m}$. (c) NRLS. (d) TV. (e) dNRLS. (f) dTV. (g) dSATV.

- Q1: In which image the microcalcifications are more conspicuous ? (Right/Left)

- Q2: In which image do you see a better representation of the breast structures ? (Right/Left)

- Q3: In which image do you see less artifacts ? Describe them. (Right/Left, then description)

- Q4: Which image do you prefer overall ? (Right/Left)

The readers had no time limitations to answer the questions.

\subsubsection{Results}

We opt for a descriptive analysis regarding the limited number of images used in this experiment. First, for each question, we show in Fig. 8 the scoring percentage in favor of dSATV and NRLS approaches pooled over all the readers. The positive end of each box corresponds to the mean-reader preference for dSATV, while the negative end corresponds to the mean-reader preference for NRLS. The top of the positive bar indicates the maximum preference score obtained over the fourteen readers for dSATV, while the top of the negative bar indicates the maximum preference score obtained over the fourteen readers for NRLS. By averaging over all the readers, our proposed method provides more conspicuous microcalcifications with $82 \%[63 \%-100 \%]$ of scores, a better 


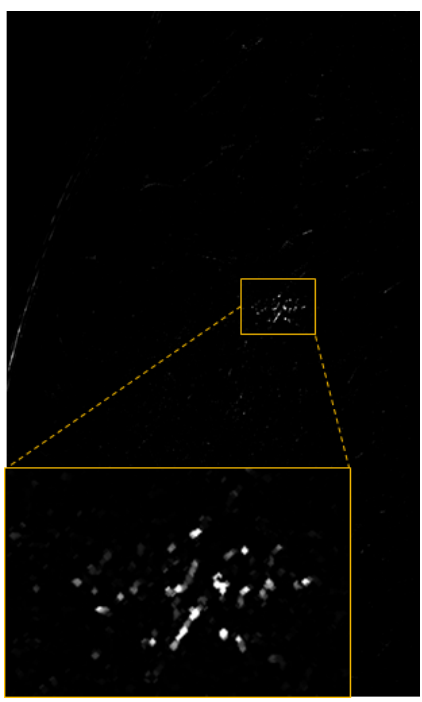

(a)

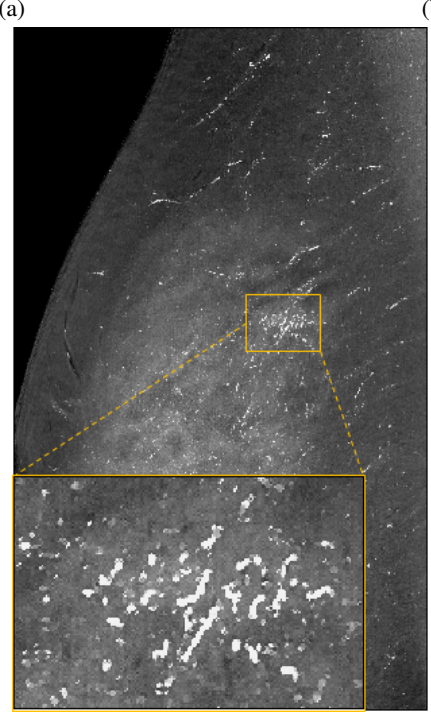

(e)

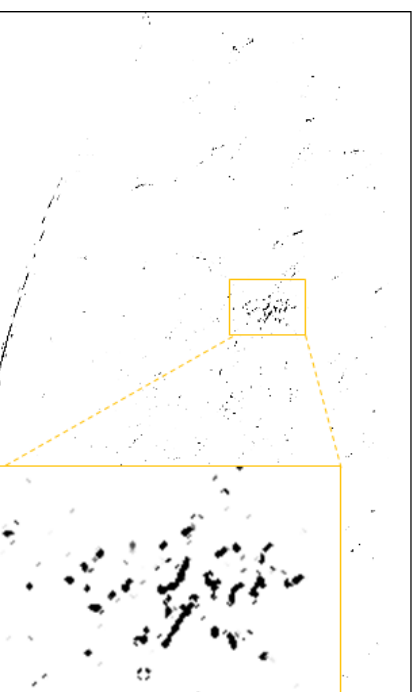

(b)

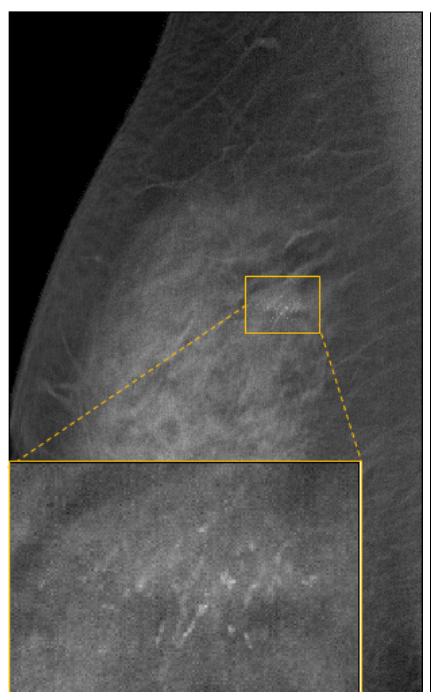

(c)

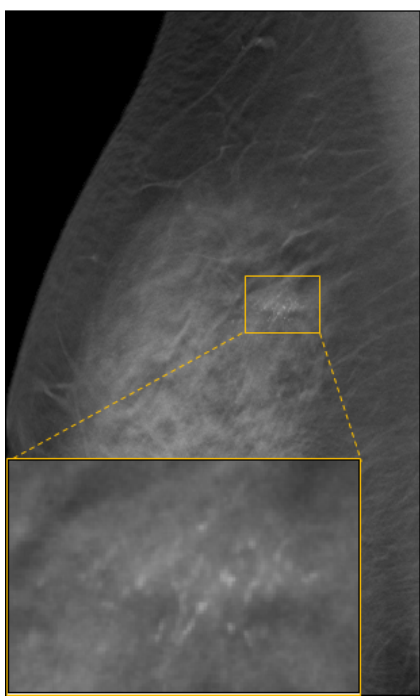

(d)

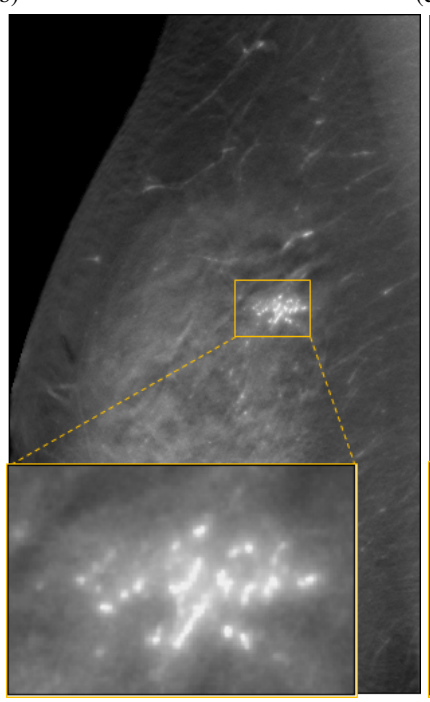

(f)

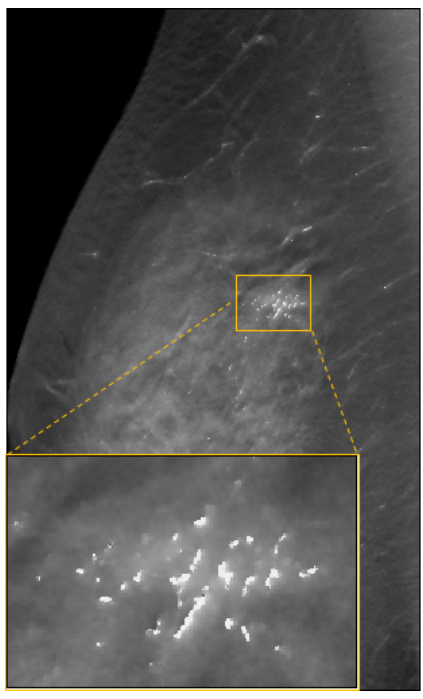

(g)

Fig. 6: Selected slice of a DBT reconstruction with size $3062 \times 994 \times 67$ (voxels) (a) Detection map $\mu$. (b) Weights $\left(\lambda_{i}\right)_{1 \leq i \leq m}$. (c) NRLS. (d) TV. (e) dNRLS. (f) dTV. (g) dSATV.

representation of the breast structures with $87 \%[58 \%-100 \%]$ of scores, less artifacts with $81 \%[47 \%-100 \%]$ of scores and an overall preference of $83 \%[58 \%-100 \%$ ] of scores. Pooling over all the fourteen readers, the most frequent artifacts observed in our proposed dSATV approach were (i) some undershooting around the microcalcifications and (ii) the presence of enhanced structures not identified as microcalcifications (false positives). Replication artifacts, presence of off-focal artifact and presence of noise were most frequently noted in the DBT images reconstructed with the non-regularized algorithm NRLS.

To assess the inter-reader agreement between all the readers, we computed the Gwet's AC1 agreement coefficient Gwet (2014) for each asked question, as displayed in Figure 9. The highest response consensus is found for breast background restoration (with a substantial inter-reader agreement) Wongpakaran et al. (2013) and the lowest one for the presence of potential artifacts (with a moderate inter-reader agreement). We notice also that the Gwet's AC1 coefficient indicates a substantial inter-reader agreement for microcalcification conspicuity and the overall preference of the DBT reconstruction in favor of dSATV. It is worthy to point out that the two latter inter-reader agreements was found to be the highest between the radiologists, while the inter-reader agreement for breast background restoration was observed to be slightly better between the GE Healthcare experts in mammography. The 


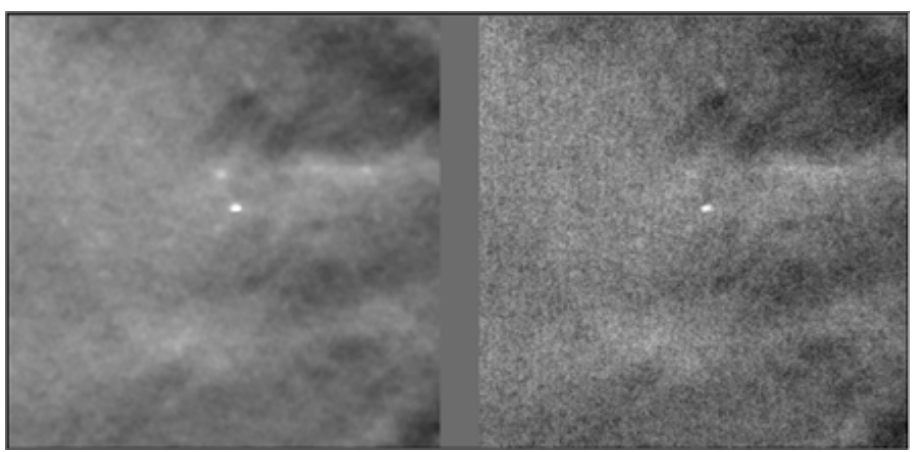

(a)

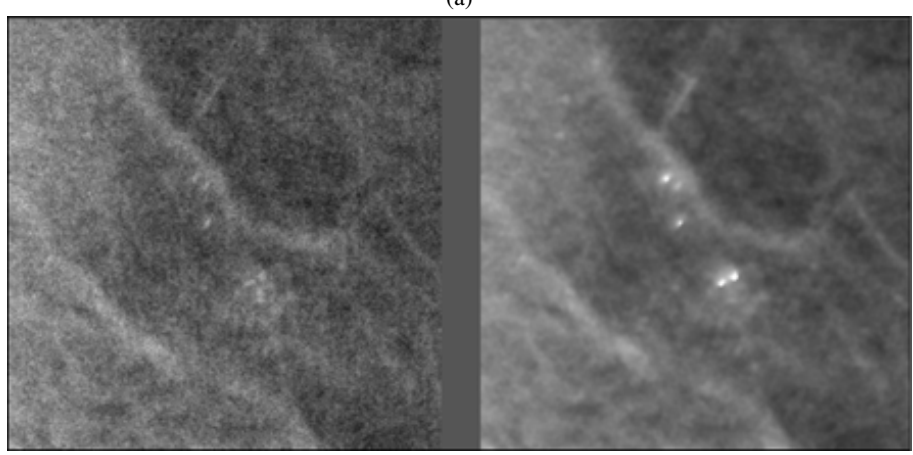

(b)

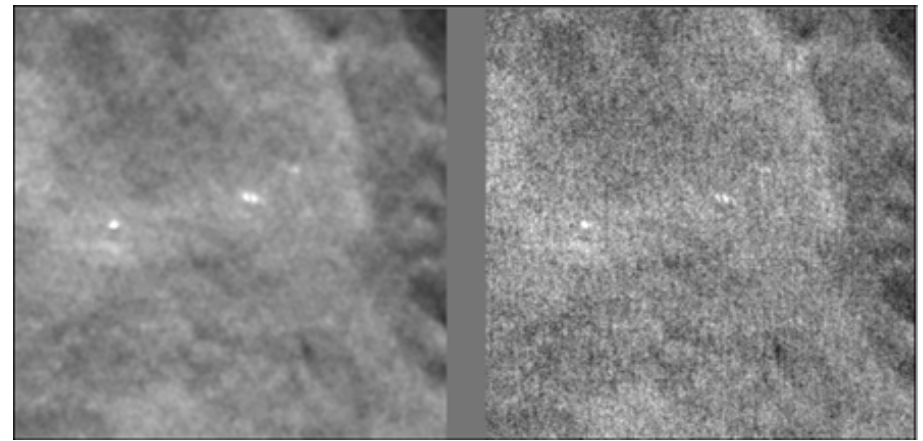

(c)

Fig. 7: Examples of slices of different image pairs used in the visual experimental study. (a) Left image : dSATV. Right image : NRLS. (b) Left image : NRLS. Right image : dSATV. (c) Left image : dSATV. Right image : NRLS.

inter-reader agreement for the observed artifacts was comparable between the two reader populations. This would tend to show that radiologists may scrutinize the DBT reconstruction in favor of the clinical task, which is directly related to the conspicuity of microcalcifications, whereas, GE Healthcare experts may be more sensitive towards the overall image quality in the DBT reconstruction.

\section{Conclusion}

We have proposed a novel reconstruction approach for DBT, which aims at improving the radiological diagnosis associated with microcalcification detection task (dSATV). The introduction of a new detectability function, an original SATV regularization function, and an improved 3MG optimization algorithm lead to an approach showing good performance on both phantom and clinical data when compared to classical DBT reconstruction techniques. The main advantage of this task-based approach is to yield more conspicuous and enhanced microcalcifications while increasing robustness to noise and improving the overall quality in the imaged volume. A visual experiment trial, conducted on fourteen experts, confirms the superiority of our proposed approach. 


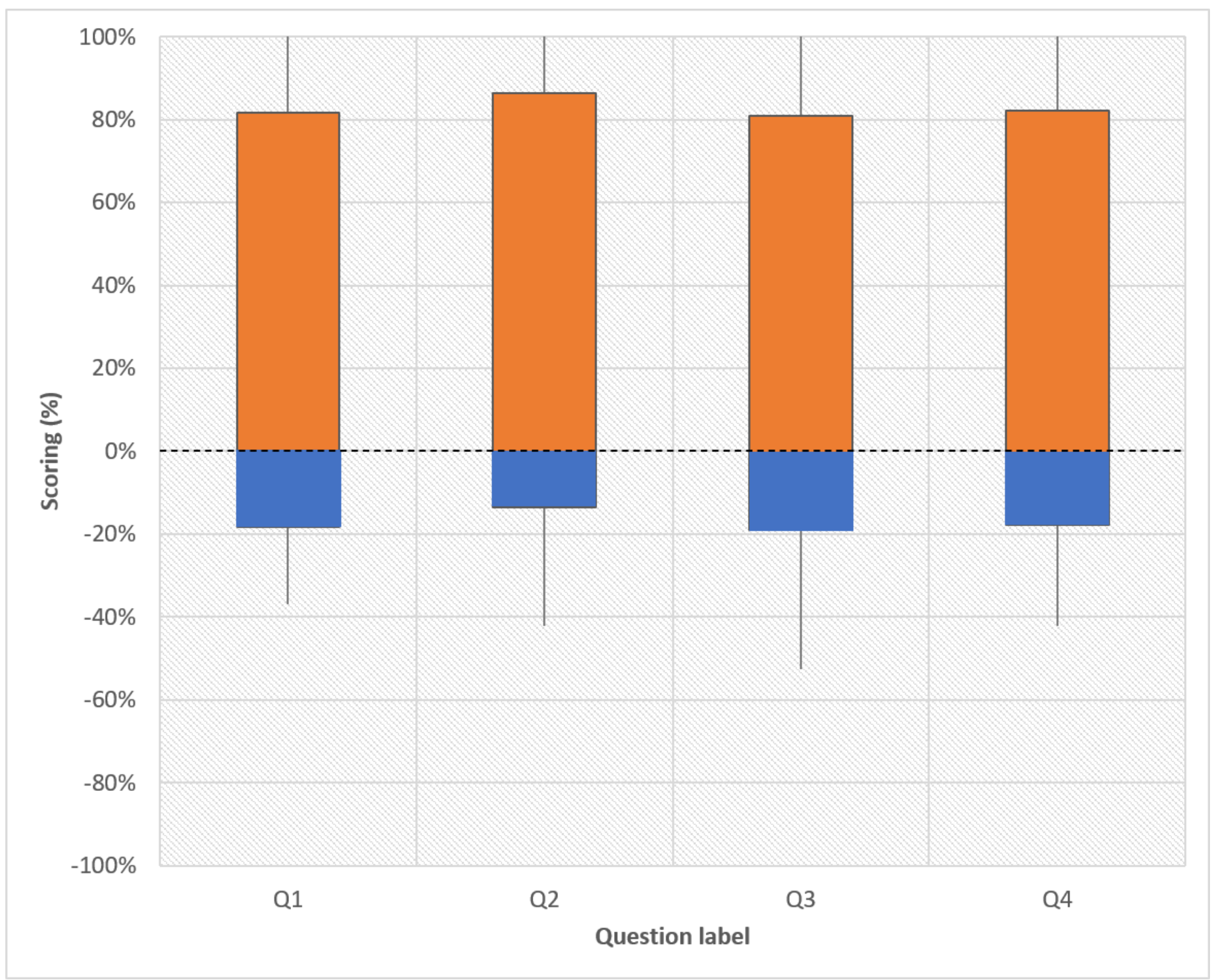

Fig. 8: Results of the visual experiment pooled over all the readers: positive scoring, displayed with orange bars, is in favor of dSATV, negative scoring (defined as positive score minus 100\%), displayed with blue bars, is in favor of NRLS.

A future direction for further improvements would be to investigate a more automatic setting of the different parameters involved in SATV, depending on the targeted task and images to be processed. Another leading idea would be to pursue the improvement of the detection map, to better distinguish between True and False Positives.

\section{Appendix A. Derivation of the detectability function using detection theory}

The detectability is defined by maximizing the log-likelihood ratio under the hypotheses (7),

$$
R=\sum_{i=1}^{q} \log \left(\frac{\mathrm{p}\left(v^{i} \mid \mathcal{H}_{1}\right)}{\mathrm{p}\left(v^{i} \mid \mathcal{H}_{0}\right)}\right)
$$

Hereabove, $\mathrm{p}\left(v^{i} \mid \mathcal{H}_{1}\right)\left(\right.$ resp. $\left.\mathrm{p}\left(v^{i} \mid \mathcal{H}_{0}\right)\right)$ designate for the probability density function of $v^{i}$ in the presence (resp. absence) of 


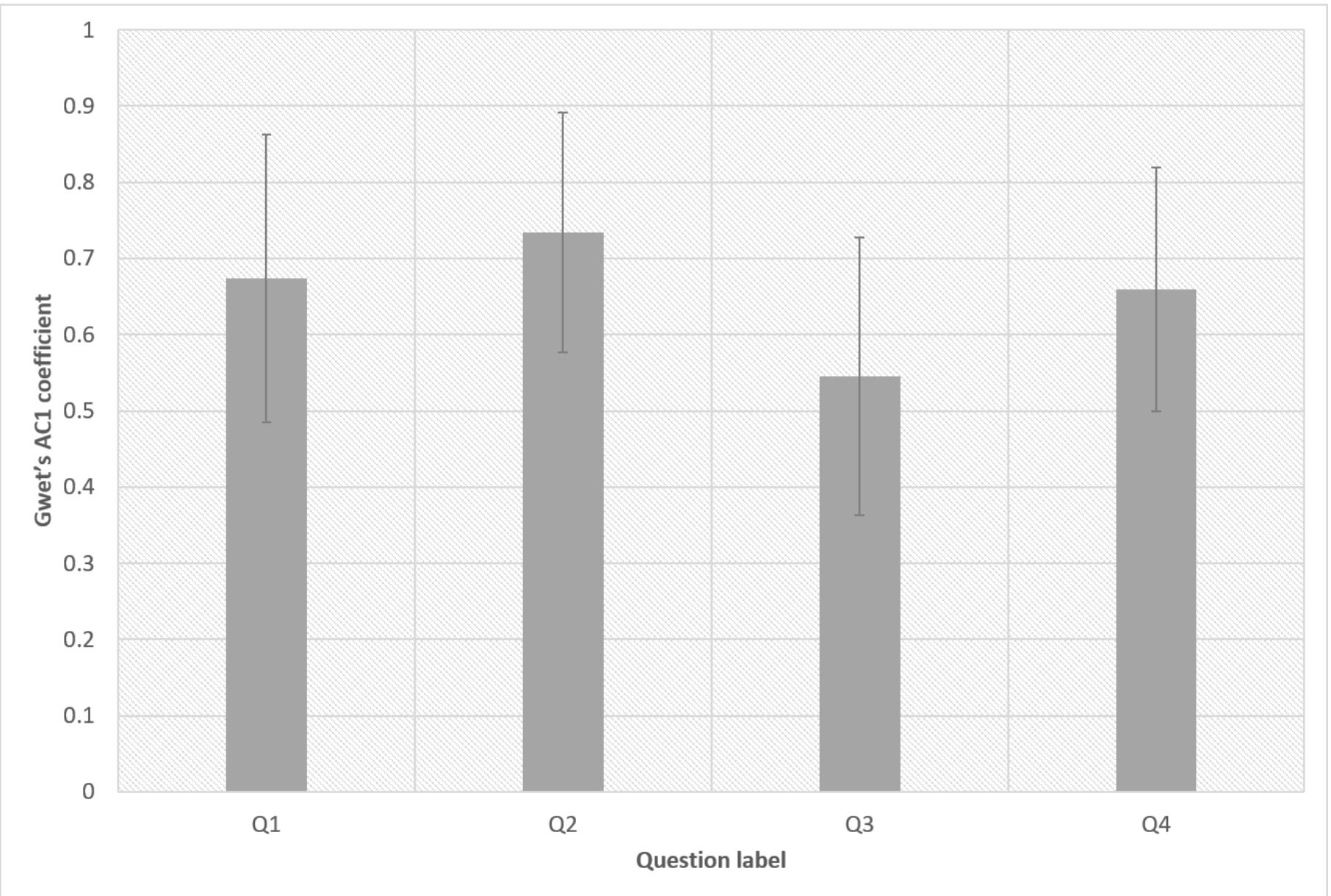

Fig. 9: Inter-reader agreement (Gwet's AC1 coefficients) between all the readers.

microcalcification, that read

$$
\begin{aligned}
& \mathrm{p}\left(v^{i} \mid \mathcal{H}_{1}\right)=\frac{1}{\sqrt{(2 \pi)^{c}|\Sigma|}} \\
& \times \exp \left(-\frac{1}{2}\left(\left(v^{i}-U^{\top}\left(b^{i}+\mu^{i}\right)\right)^{\top} \Sigma^{-1}\left(v^{i}-U^{\top}\left(b^{i}+\mu^{i}\right)\right)\right)\right), \\
& \mathrm{p}\left(v^{i} \mid \mathcal{H}_{0}\right)=\frac{1}{\sqrt{(2 \pi)^{c}|\Sigma|}} \\
& \times \exp \left(-\frac{1}{2}\left(\left(v^{i}-U^{\top} b^{i}\right)^{\top} \Sigma^{-1}\left(v^{i}-U^{\top} b^{i}\right)\right)\right) .
\end{aligned}
$$

In order to compute the latter matrix, we need to estimate the sample means of the feature vectors $v^{i}$ conditioned to both hypotheses. This enables the computation of the maximum likelihood ratio $R$ in $(A .1)$. Now, based on the expression (7), we are able to calculate it as

$$
R=\sum_{i=1}^{q} \log \mathrm{p}\left(v^{i} \mid \mathcal{H}_{1}\right)-\log \mathrm{p}\left(v^{i} \mid \mathcal{H}_{0}\right) .
$$


By using the expressions of the probability density functions in (A.2), we obtain, after some simplifications,

$$
\begin{aligned}
& R=\sum_{i=1}^{q}-\frac{1}{2}\left(v^{i}-U^{\top}\left(b^{i}+\mu^{i}\right)\right)^{\top} \Sigma^{-1}\left(v^{i}-U^{\top}\left(b^{i}+\mu^{i}\right)\right) \\
& +\frac{1}{2}\left(v^{i}-U^{\top} b^{i}\right)^{\top} \Sigma^{-1}\left(v^{i}-U^{\top} b^{i}\right) \\
& =\sum_{i=1}^{q} \frac{1}{2}\left(\left(b^{i}+\mu^{i}\right)^{\top} U \Sigma^{-1} v^{i}+\left(v^{i}\right)^{\top} \Sigma^{-1} U^{\top}\left(b^{i}+\mu^{i}\right)\right) \\
& -\frac{1}{2}\left(\left(b^{i}\right)^{\top} U \Sigma^{-1} v^{i}-\left(v^{i}\right)^{\top} \Sigma^{-1} U^{\top} b^{i}+\left(\mu^{i}\right)^{\top} U \Sigma^{-1} U^{\top} \mu^{i}\right) .
\end{aligned}
$$

The fact that $\Sigma^{-1}$ is a symmetric matrix leads to the following simplification:

$$
R=\sum_{i=1}^{q}\left(\mu^{i}\right)^{\top} U \Sigma^{-1} U^{\top} r^{i}-\frac{1}{2}\left(\mu^{i}\right)^{\top} U \Sigma^{-1} U^{\top} \mu^{i} .
$$

Since the $\mu^{i}$, s are fixed, the quantity to be maximized using 6 is

$$
\begin{aligned}
\left(\forall d \in \mathbb{R}^{m}\right) \quad D(d) & =\sum_{i=1}^{q}\left(\mu^{i}\right)^{\top} U \Sigma^{-1} U^{\top} S_{i} d \\
& =\sum_{i=1}^{q} \sum_{j=1}^{q}\left(\mu^{j}\right)^{\top} S_{j} S_{i}^{\top} U \Sigma^{-1} U^{\top} S_{i} d \\
& =\mu^{\top} \sum_{i=1}^{q} S_{i}^{\top} U \Sigma^{-1} U^{\top} S_{i} d
\end{aligned}
$$

where $\mu$ is given by 9 . The last equality has been derived from the properties of the operators $\left(S_{i}\right)_{1 \leq i \leq q}$ and the assumption that the ROIs do not overlap, which translate into the following properties:

$$
\left(\forall(i, j) \in\{1, \ldots, q\}^{2}\right) \quad S_{j} S_{i}^{\top}= \begin{cases}I_{k} & \text { if } j=i \\ 0 & \text { otherwise }\end{cases}
$$

This yields our microcalcification detectability function designed for any reconstructed volume $d \in \mathbb{R}^{m}$.

\section{Appendix B. Estimation of $\Sigma$}

Based on hypotheses $\mathcal{H}_{0}$ and $\mathcal{H}_{1}$. the training dataset is divided into two subsets $F_{0}$ and $F_{1}$ with cardinalities $t_{0}$ and $t_{1}$ respectively:

$$
\begin{aligned}
& F_{0}=\left\{\tilde{r}_{\mid \mathcal{H}_{0}}^{j} \in \mathbb{R}^{k} \mid j \in\left\{1, \ldots, t_{0}\right\}\right\} \\
& F_{1}=\left\{\tilde{r}_{\mid \mathcal{H}_{1}}^{j} \in \mathbb{R}^{k} \mid j \in\left\{1, \ldots, t_{1}\right\}\right\} .
\end{aligned}
$$

We can then infer, for every $\ell \in\{0,1\}$ and $j \in\left\{1, \ldots, t_{\ell}\right\}$, the channelized image as follows:

$$
\tilde{v}_{\mid \mathcal{H}_{\ell}}^{j}=U^{\top} \tilde{r}_{\mid \mathcal{H}_{\ell}}^{j}
$$

The estimation of the covariance matrix $\Sigma$ of the channelized noise is deduced as

$$
\Sigma=\frac{1}{t_{0}+t_{1}} \sum_{\ell=0}^{1} \sum_{j=1}^{t_{\ell}}\left(\tilde{v}_{\mid \mathcal{H}_{\ell}}^{j}-\overline{\tilde{v}}_{\mid \mathcal{H}_{\ell}}\right)\left(\tilde{v}_{\mid \mathcal{H}_{\ell}}^{j}-\overline{\tilde{v}}_{\mid \mathcal{H}_{\ell}}\right)^{\top},
$$

where

$$
\overline{\tilde{v}}_{\mid \mathcal{H}_{\ell}}=\frac{1}{t_{\ell}} \sum_{j=1}^{t_{\ell}} \tilde{v}_{\mid \mathcal{H}_{\ell}}^{j}, \quad \ell \in\{0,1\}
$$




\section{Appendix C. Primal-dual solution to Problem (10)}

For the resolution of Problem (10), we propose to employ Algorithm 2, where $\mathrm{P}_{C}$ denotes the projection onto a nonempty closed convex set $C$ and $J_{\max }>0$ is the total number of iterations.

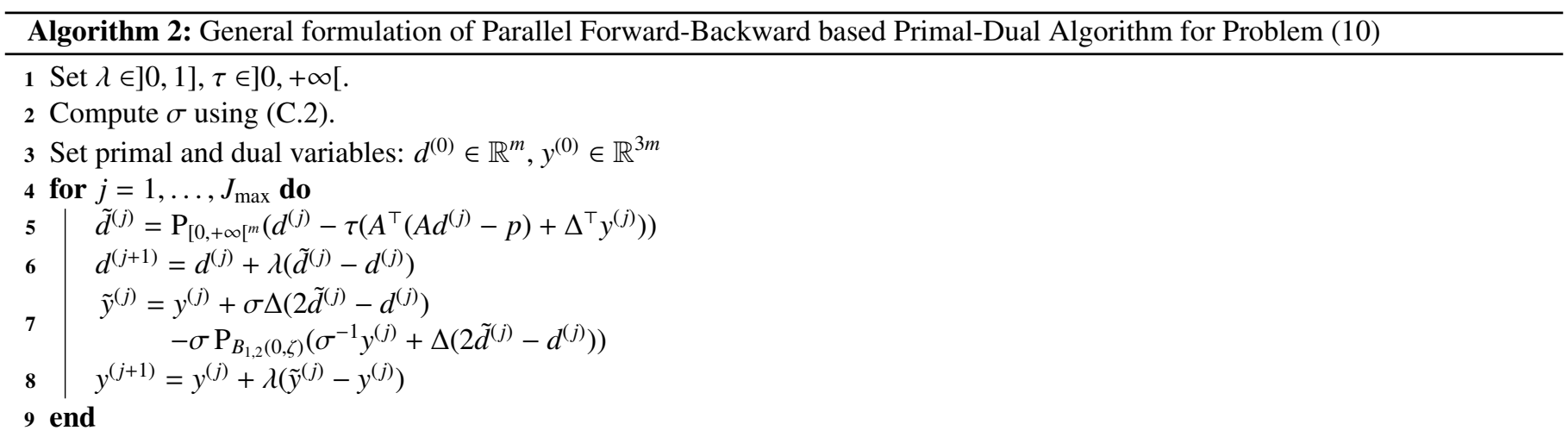

The algorithm parameter $(\sigma, \tau)$ are chosen so as to satisfy the theoretical requirements in Condat (2013); Vũ (2013) in order to guarantee the convergence of Algorithm 2 .

$$
\tau^{-1}-\sigma\|\Delta\|^{2} \geq \frac{1}{2\|A\|^{2}}
$$

where $\|A\|$ (resp. $\|\Delta\|)$ denotes the spectral norm of $A$ (resp. $\Delta$ ). To set the algorithm parameters in practice, we simply fix $\tau \in] 0,2\|A\|^{2}[$ and compute $\sigma$ as follows:

$$
\sigma=\frac{1}{\|\Delta\|^{2}}\left(\tau^{-1}-\frac{1}{2\|A\|^{2}}\right)
$$

\section{Appendix D. Detailed derivations of 3MG algorithm}

The $3 \mathrm{MG}$ algorithm Chouzenoux et al. (2013b) consists of building a sequence $\left(d^{(j)}\right)_{j \in \mathbb{N}} \in \mathbb{R}^{m}$ of approximation to the sought volume so as to minimize function 20 . The $3 \mathrm{MG}$ iterates are defined as

$$
(\forall j \in \mathbb{N}) \quad d^{(j+1)}=d^{(j)}+B^{(j)} u^{(j)}
$$

$d^{(0)} \in \mathbb{R}^{m}$ is an initial volume, $B^{(j)} \in \mathbb{R}^{m \times 2}$ stacks the set of memory gradient directions:

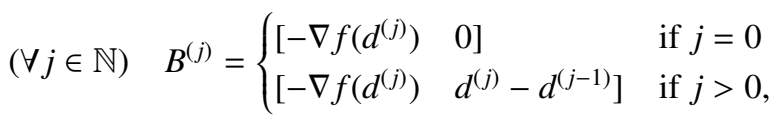

and $u^{(j)} \in \mathbb{R}^{2}$ is a stepsize designed to minimize a quadratic majorizing approximation for $u \mapsto f\left(d^{(j)}+B^{(j)} u\right)$.

Let $d^{\prime} \in \mathbb{R}^{m}$. A function $q\left(., d^{\prime}\right)$ is said to be a tangent majorant for $f$ at $d^{\prime}$, if for every $d \in \mathbb{R}^{m}$,

$$
q\left(d, d^{\prime}\right) \geq f(d) \quad \text { and } \quad q\left(d^{\prime}, d^{\prime}\right)=f\left(d^{\prime}\right)
$$

Starting from the construction of the tangent majorant $q$, at each iteration $j \in \mathbb{N}$, the step size $u^{(j)}$ is then obtained by minimizing the quadratic function $u \mapsto q\left(d^{(j)}+B^{(j)} u, d^{(j)}\right)$, which yields the following closed form expression:

$$
u^{(j)}=-\left(\left(B^{(j)}\right)^{\top} \mathcal{M}\left(d^{(j)}\right) B^{(j)}\right)^{\dagger}\left(B^{(j)}\right)^{\top} \nabla f\left(d^{(j)}\right)
$$

where $\dagger$ denotes the pseudo-inverse operation. The 3MG algorithm iterates over Steps (D.2), (D.4), and (D.1). The convergence of the sequence $\left(d^{(j)}\right)_{j \in \mathbb{N}}$ produced by $3 \mathrm{MG}$ to a minimizer of $f$ is secured Chouzenoux et al. (2013a). 


\section{Acknowledgments}

We are thankful to Dr Ann-Katherine Carton for her expert support in the implementation and statistical analysis of the preference trial.

This work was partially founded by ANRT grant n²017/0106. Emilie Chouzenoux and Jean-Christophe Pesquet acknowledge support from the European Research Council Starting Grant MAJORIS ERC-2019-STG-850925 and the ANR Research and Teaching Chair BRIDGEABLE in Artificial Intelligence, respectively.

\section{References}

Abergel, R., Moisan, L., 2017. The shannon total variation. J. Math. Imag. Vision 59, 341-370.

Aujol, J., 2009. Some first-order algorithms for total variation based image restoration. J. Math. Imag. Vision 34, 307-327.

Barrett, H., Yao, J., Rollandt, J., Myerst, K., 1993. Model observers for assessment of image quality. Proc. Natl. Acad. Sci. 90, 9758-9765.

Berger, C., Geraud, T., Levillain, R., Widynski, N., Baillard, A., Bertin, E., 2007. Effective Component Tree Computation with Application to Pattern Recognition in Astronomical Imaging, in: Proc. IEEE Int. Conf. Image Process. (ICIP 2007), San Antonio, TX. pp. 41-44. doi 10.1109/ICIP. 2007.4379949

Biton, S., Gilboa, G., 2018. Adaptive anisotropic total variation - a nonlinear spectral analysis. arXiv preprint arXiv:1811.11281 .

Blomgren, P., Chan, T.F., Mulet, P., Wong, C., 1997. Total variation image restoration: numerical methods and extensions, in: Proc. Int. Conf. Image Process. (ICIP 1997), IEEE. pp. 384-387.

Boyd, S., Parikh, N., Chu, E., Peleato, B., Eckstein, J., 2011. Distributed optimization and statistical learning via the alternating direction method of multipliers. Found. Trends Mach. Learn. 3, 1-122.

Calamai, P.H., Moré, J.J., 1987. Projected gradient methods for linearly constrained problems. Math. Prog. 39, 93-116.

Chambolle, A., Dossal, C., 2015. On the convergence of the iterates of "fista". J. Optim. Theory Appl. 166, 25.

Chambolle, A., Levine, S., Lucier, B., 2011. An upwind finite-difference method for total variation-based image smoothing. SIAM J. Imaging Sciences 4, $277-299$.

Chan, T., Esedoglu, S., Park, F., 2007. Image decomposition combining staircase reduction and texture extraction. J. Visual Comm. Image Repres. 18, $464-486$.

Chan, T., Marquina, A., Mulet, P., 2000. High-order total variation-based image restoration. SIAM J. Sci. Comput. 22, 503-516.

Chierchia, G., Pustelnik, N., Pesquet-Popescu, B., Pesquet, J., 2014. A nonlocal structure tensor-based approach for multicomponent image recovery problems. IEEE Trans. Image Processing 23, 5531-5544.

Chouzenoux, E., Jezierska, A., Pesquet, J.C., Talbot, H., 2013a. A majorize-minimize subspace approach for $\ell_{2}-\ell_{0}$ image regularization. SIAM J. Imaging Sciences $6,563-591$.

Chouzenoux, E., Pesquet, J.C., 2016. Convergence rate analysis of the majorize-minimize subspace algorithm. IEEE Signal Process. Lett. 23, $1284-1288$.

Chouzenoux, E., Pesquet, J.C., 2017. A stochastic majorize-minimize subspace algorithm for online penalized least squares estimation. IEEE Trans. Signal Processing 65, 4770-4783.

Chouzenoux, E., Zolyniak, F., Gouillart, E., Talbot, H., 2013b. A majorize-minimize memory gradient algorithm applied to x-ray tomography, in: Proc. IEEE Int. Conf. Image Process. (ICIP 2013), IEEE, Melbourne, Australia. pp. 1011-1015.

Christoyianni, I., Koutras, A., Dermatas, E., Kokkinakis, G., 2002. Computer aided diagnosis of breast cancer in digitized mammograms. Comput. Med. Imaging Graph. 26, 309 - 319. URL: http://www.sciencedirect.com/science/article/pii/S0895611102000319 doi https://doi.org/10.1016/ S0895-6111(02) 00031-9

Combettes, P., Pesquet, J., 2011. Proximal splitting methods in signal processing, in: Fixed-point algorithms for inverse problems in science and engineering. Springer, pp. 185-212.

Combettes, P.L., Pesquet, J.C., 2021. Fixed point strategies in data science. IEEE Trans. Sig. Proc. 69, 3878-3905.

Condat, L., 2013. A primal-dual splitting method for convex optimization involving Lipschitzian, proximable and linear composite terms. J. Optim. Theory Appl. $158,460-479$.

Condat, L., 2017. Discrete total variation: New definition and minimization. SIAM J. Imaging Sciences 10, 1258-1290.

Corbineau, M.C., Chouzenoux, E., Pesquet, J.C., 2018. Geometry-texture decomposition/reconstruction using a proximal interior point algorithm, in: Proc. IEEE Sensor Array Multichannel Signal Process. Work. (SAM), IEEE. pp. 435-439.

Dang, H., Stayman, J., Xu, J., Zbijewski, W., Sisniega, A., Mow, M., Wang, X., Foos, D., Aygun, N., Koliatsos, V., Siewerdsen, J., 2017. Task-based statistical image reconstruction for high-quality cone-beam ct. Phys. Med. Biol. 62, 8693-8719. URL: http://stacks . iop. org/0031-9155/62/i=22/a=8693

De Pierro, A.R., 1995. A modified expectation maximization algorithm for penalized likelihood estimation in emission tomography. IEEE Trans. Medical Imaging $14,132-137$.

Destounis, S., Arieno, A., Morgan, R., 2013. Preliminary clinical experience with digital breast tomosynthesis in the visualization of breast microcalcifications. J. Clin. Imaging Sci. 3.

Erdogan, H., Fessler, J.A., 1999. Ordered subsets algorithms for transmission tomography. Physics in Medicine and Biology 44, 2835-2851. URL: https: //doi.org/10.1088\%2F0031-9155\%2F44\%2F11\%2F311 doi 10.1088/0031-9155/44/11/311

Florescu, A., Chouzenoux, E., Pesquet, J.C., Ciuciu, P., Ciochina, S., 2014. A majorize-minimize memory gradient method for complex-valued inverse problem. Signal Process. 103, 285-295. Special issue on Image Restoration and Enhancement: Recent Advances and Applications.

Gwet, K.L., 2014. Handbook of inter-rater reliability: The definitive guide to measuring the extent of agreement among raters. Advanced Analytics, LLC.

Henrot, P., Leroux, A., Barlier, C., Génin, P., 2014. Breast microcalcifications: the lesions in anatomical pathology. Diagn. Interv. Imag. 95, 141-152.

Horvat, J.V., Keating, D.M., Rodrigues-Duarte, H., Morris, E.A., Mango, V.L., 2019. Calcifications at digital breast tomosynthesis: Imaging features and biopsy techniques. Radiographics 39.

Jacobson, M.W., Fessler, J.A., 2007. An expanded theoretical treatment of iteration-dependent majorize-minimize algorithms. IEEE Trans. Image Processing 16, 2411-2422.

Kolb, T., Lichy, J., Newhouse, J., 2002. Comparison of the performance of screening mammography, physical examination, and breast us and evaluation of factors that influence them: an analysis of 27,825 patient evaluations. Radiology 225, 165-175.

Komodakis, N., Pesquet, J., 2014. Playing with Duality : An Overview of Recent Primal-Dual Approaches for Solving Large-Scale Optimization Problems. IEEE Signal Processing Mag. 32, $31-54$.

Kopans, D., Gavenonis, S., Halpern, E., Moore, R., 2011. Calcifications in the breast and digital breast tomosynthesis. Breast J. 17, 638-644. 
Kuwabara, N., Takuwa, H., Takeuchi, M., Kawashima, H., 2020. Can digital breast tomosynthesis improve identification of malignant calcifications? Radiol. Phys. Technol. 13, 249-255.

Li, J., Zhang, H., Jiang, H., Guo, X., Zhang, Y., Qi, D., Guan, J., Liu, Z., Wu, E., Luo, S., 2018a. Diagnostic performance of digital breast tomosynthesis for breast suspicious calcifications from various populations: A comparison with full-field digital mammography. Comput. Struct. Biotechnol. J. $17,82-89$.

Li, Z., Desolneux, A., Muller, S., Milioni de Carvalho, P., Carton, A., 2018b. Comparison of microcalcification detectability in FFDM and DBT using a virtual clinical trial, in: Proc. SPIE Med. Imaging, Houston, Texas. URL: https://doi.org/10.1117/12.2293619 doi 10.1117/12.2293619

Liao, F., Shao, S., 2017. An image denoising fast algorithm for weighted total variation, in: Proc. Int. Conf. Intell. Information Process., ACM. p. 16.

Louchet, C., Moisan, L., 2008. Total variation denoising using posterior expectation, in: Proc. Eur. Signal Process. Conf. (EUSIPCO 2008), IEEE. pp. 1-5.

Luo, F., Li, W., Tu, W., Wu, W., 2018. Adaptive weighted total variation minimization based alternating direction method of multipliers for limited angle CT reconstruction. IEEE Access 6, 64225-64236.

Merveille, O., Miraucourt, O., Salmon, S., Passat, N., Talbot, H., 2016. A variational model for thin structure segmentation based on a directional regularization, in: Proc. IEEE Int. Conf. Image Process. (ICIP 2016), IEEE. pp. 4324-4328.

Metin, E., Isa, Y., Mustafa, K., Aydin, A., 2014. An iterative tomosynthesis reconstruction using total variation combined with non-local means filtering. BioMed. Eng. OnLine 13, 65. URL: https://doi.org/10.1186/1475-925X-13-65 doi 10.1186/1475-925X-13-65

Michell, M., Iqbal, A., Wasan, R., Evans, D., Peacock, C., Lawinski, C., Douiri, A., Wilson, R., Whelehan, P., 2012. A comparison of the accuracy of film-screen mammography, full-field digital mammography, and digital breast tomosynthesis. Clin. Radiol. 67, 976-981.

Myers, K., Barrett, H., 1987. Addition of a channel mechanism to the ideal-observer model. J. Opt. Soc. Am. (A) 4, $2447-2457$.

Nikolova, M., 2000. Local strong homogeneity of a regularized estimator. SIAM J. Appl. Mathematics 61, 633-658.

Park, S., Barrett, H., Clarkson, E., Kupinski, M., Myers, K., 2007. Channelized-ideal observer using Laguerre-Gauss channels in detection tasks involving nonGaussian distributed lumpy backgrounds and a Gaussian signal. J. Opt. Soc. Am. (A) 24, B136-B150. URL: http://josaa.osa.org/abstract.cfm?URI= josaa-24-12-B136 doi $10.1364 /$ JOSAA.24.00B136

Park, S., Witten, J.M., Myers, K.J., 2009. Singular vectors of a linear imaging system as efficient channels for the bayesian ideal observer. IEEE Trans. Medical Imaging 28.5, 657-668. doi 10.1109/TMI .2008.2008967

Piccolomini, E.L., Morotti, E.L., 2016. A fast Total Variation-based iterative algorithm for digital breast tomosynthesis image reconstruction 10, 277-289. doi doi : 10.1177/1748301816668022

Platiša, L., Goossens, B., Vansteenkiste, E., Park, S., Gallas, B., Badano, A., Philips, W., 2011. Channelized Hotelling observers for the assessment of volumetric imaging data sets. J. Opt. Soc. Am. (A) 28, 1145-1163. URL: http://josaa.osa.org/abstract.cfm?URI=josaa-28-6-1145 doi 10.1364/JOSAA . 28.001145

Poplack, S., Tosteson, T., Kogel, C., Nagy, H., 2007. Digital breast tomosynthesis: initial experience in 98 women with abnormal digital screening mammography. Am. J. Roentgenol. 189, 616-623.

Pustelnik, N., Benazza-Benhayia, A., Zheng, Y., Pesquet, J., 2016. Wavelet- based image deconvolution and reconstruction. Wiley Encyclopedia of Electrical and Electronics Engineering, 1-34doi 10.1002/047134608X.W8294

American College of Radiology, A.C.R., D’Orsi, C.J., et al., 2013. ACR BI-RADS Atlas: Breast Imaging Reporting and Data System; Mammography, Ultrasound, Magnetic Resonance Imaging, Follow-up and Outcome Monitoring, Data Dictionary. ACR, American College of Radiology.

Rose, S., Sanchez, A., Sidky, E., Pan, X., 2017. Investigating Simulation-Based Metrics for Characterizing Linear Iterative Reconstruction in Digital Breast Tomosynthesis. Med. Phys. 44, 279-296.

Rose, S.D., Sidky, E.Y., Reiser, I., Pan, X., 2019. Imaging of fiber-like structures in digital breast tomosynthesis. J. Med. Imaging 6, 031404.

Sechopoulos, I., 2013a. A review of breast tomosynthesis. Part I . The image acquisition process. Med. Phys. 40, 1-12.

Sechopoulos, I., 2013b. A review of breast tomosynthesis. Part II. Image reconstruction, processing and analysis, and advanced applications. Med. Phys. $40,014302$.

Sidky, E., Pan, X., Reiser, I., Nishikawa, R., Moore, R., Kopans, D., 2009. Enhanced imaging of microcalcifications in digital breast tomosynthesis through improved image-reconstruction algorithms. Med. Phys. 36, 4920-4932. URL: http://www.pubmedcentral.nih.gov/articlerender.fcgi?artid= $2773453\{\&\}$ tool=pmcentrez $\{\&\}$ rendertype=abstract doi $10.1118 / 1.3232211$

Sidky, E., Reiser, I., Nishikawa, R., Pan, X., 2007. Image reconstruction in digital breast tomosynthesis by total variation minimization, in: Proc. SPIE Med. Imag., San Diego, CA.

Spangler, M., Zuley, M., Sumkin, J., Abrams, G., Ganott, M., Hakim, C., Perrin, R., Chough, D., Shah, R., Gur, D., 2011. Detection and classification of calcifications on digital breast tomosynthesis and 2d digital mammography: a comparison. Am. J. Roentgenol. 196, 320-324.

Sun, Y., Babu, P., Palomar, D.P., 2017. Majorization-minimization algorithms in signal processing, communications, and machine learning. IEEE Trans. Signal Processing 65, 794-816.

Szasz, T., Basarab, A., Kouamé, D., 2016. Beamforming through regularized inverse problems in ultrasound medical imaging. IEEE Trans. Ultrason. Ferroelectr. Freq. Control 63, 2031-2044.

Vũ, B.C., 2013. A splitting algorithm for dual monotone inclusions involving cocoercive operators. Adv. Comput. Math. 38, 667-681.

Van Metter, R., Beutel, J., Kundel, H.L., 2000. Handbook of Medical Imaging. volume 1. Laurel, MD.

Wheeler, F., Perera, A., Claus, B., Muller, S., Peters, G., Kaufhold, J., 2006. Micro-calcification detection in digital tomosynthesis mammography, in: Proc. SPIE Med. Imaging, San Diego, CA. URL: https://doi.org/10.1117/12.653478 doi doi:10.1117/12.653478

Wongpakaran, N., Wongpakaran, T., Wedding, D., Gwet, K.L., 2013. A comparison of cohen's kappa and gwet's ac1 when calculating inter-rater reliability coefficients: a study conducted with personality disorder samples. BMC medical research methodology 13, 61 .

Xin, H., Park, S., 2013. Model observers in medical imaging research. Theranostics 3, 774.

Xu, G., Xia, Y., Ji, H., 2018. Weighted total variation based convex clustering. arXiv preprint arXiv:1808.09144 . 